\title{
Coherent-radiation-induced longitudinal single-pass beam breakup instability of a steady-state microbunch train in an undulator
}

\author{
Cheng-Ying Tsai॰* \\ School of Electrical and Electronic Engineering, Huazhong University of Science and Technology, \\ Wuhan 430074, China \\ Alexander Wu Chao \\ Stanford University, Stanford, California 94309, USA \\ Yi Jiao \\ Key Laboratory of Particle Acceleration Physics and Technology, Institute of High Energy Physics, \\ Chinese Academy of Sciences, Beijing 100049, China \\ and University of Chinese Academy of Sciences, Beijing 100049, China \\ Hao-Wen Luo \\ Department of Engineering and System Science, National Tsing Hua University, \\ Hsinchu 30013, Taiwan \\ and National Synchrotron Radiation Research Center, 101 Hsin-Ann Road, Hsinchu 30076, Taiwan \\ Make Ying $\odot$ \\ Institute of Photonics Technologies and Department of Electrical Engineering, \\ National Tsing Hua University, Hsinchu 30013, Taiwan \\ Qinghong Zhou \\ School of Science, Southwest University of Science and Technology, Mianyang, Sichun 621010, China
}

(Received 22 August 2021; accepted 1 November 2021; published 29 November 2021)

It has been known that a high-brightness electron beam emits broadband synchrotron radiation when traversing a curved orbit. The radiation reaction at wavelengths comparable to the bunch length or to the wavelength of a phase space modulated beam may lead to collective instabilities. In this paper, we investigate a potential single-pass instability mechanism of coherent-radiation-induced longitudinal multibunch beam breakup (BBU) instability in the presence of a closely spaced microbunch train in an undulator, particularly when the microbunch spacing is close to the resonant wavelength of the undulator. This problem is formulated based on the macroparticle model together with the slippage constraint on the beam-wave interaction. The set of coupled differential equations for individual microbunches can be solved analytically for the first few microbunches with linearization of the coherent radiation wakefield, and numerically in general nonlinear cases for unequal spacing or nonuniform filling charges. The underlying mechanisms, including the slippage effect, the potential-well effect leading to extra focusing, dependence of microbunch spacing and filling patterns, are discussed. The analysis is then applied to the recently proposed steady-state microbunching (SSMB) mechanism with two examples serving for the high average coherent radiation power sources for extreme ultraviolet (EUV) and infrared wavelength regions. For the specific scenario considered in this paper, it is found that when the microbunch spacing is close to the fundamental resonant wavelength, the coherent radiation can provide extra longitudinal focusing for the individual microbunches, leading to more stable multibunch oscillations. For the preliminary nominal SSMB designs with the average beam current of $1 \mathrm{~A}$, our studies show that the single-pass longitudinal BBU instability should not be a severe issue.

DOI: 10.1103/PhysRevAccelBeams.24.114401

\footnotetext{
*jcytsai@ hust.edu.cn
}

Published by the American Physical Society under the terms of the Creative Commons Attribution 4.0 International license. Further distribution of this work must maintain attribution to the author(s) and the published article's title, journal citation, and DOI. 


\section{INTRODUCTION}

Beam quality preservation is of general concern in delivering a high-brightness electron beam through a beam transport line, recirculation arc or storage ring in the design and operation of modern particle accelerators. A highbrightness beam is usually characterized by low emittance, small uncorrelated energy spread, short bunch length and/or high bunch charge. When interacting electromagnetically with the environment, such a high-brightness beam is subject to collective instabilities [1-3]. Typical collective instabilities consist of short range single-bunch instabilities, e.g., beam breakup (BBU) instability (see Refs. [4-7] for a few examples), head-tail instability [8,9], microbunching instability (MBI) in single-pass [10-14], storage ring [15-18], and recirculating accelerators $[19,20]$. Depending on how fast the beam-induced electromagnetic fields decay, other situations for longrange instabilities include single-pass multibunch, multipass single-bunch, or multipass multibunch instabilities (for more detailed introduction, see Refs. [1,2] and references therein). In this paper, we aim to study the single-pass multibunch instability. Here we note that, when a beam is subject to an instability, it may lead to beam quality degradation, e.g., emittance growth and/or energy spread increase, and in some serious situations may even cause partial or total particle losses, all eventually resulting in machine performance limitation.

The collective effects can involve beam-environment interaction with localized objects, e.g., the wake fields generated by a source particle passing through a cavity-like structure [1-3]. Depending on the decay rate of the wake, the energy kick on the following test particles can be either in the same bunch as the one the source particle lies in, or in the following bunches. The collective interaction may also occur via co-propagating, synchrotron radiation mechanism, e.g., the coherent synchrotron radiation (CSR) [21-29]. The CSR fields originate from the situation that an electron beam traversing a bending magnet emits a broadband radiation. The beam particles travel along a curve orbit, while the radiation fields propagate in straight lines in free space at the speed of light. When the retardation condition is fulfilled for the source particles (which emitted radiation) and the test particles (which receives radiation), the electrons acquire an energy kick, leading to the so-called tail-head interaction [21]. The corresponding radiation wavelength can cover the range comparable to the electron bunch length or the length of the internal (modulated) structures of an electron beam. Therefore, the correlated energy kicks can be transformed to density modulations downstream in longitudinal dispersive regions. Such a conversion of density-energy modulations, if forming a positive feedback, can lead to enhancement of modulation amplitudes. This collective interaction, known as MBI, has been studied theoretically and confirmed experimentally in various accelerator facilities in the past two decades [10-20]. It has been found that CSR-induced MBI can have a significant impact on the high-brightness beam along a transport line consisting of a series of bending magnets [30-33]. It may also impose a challenging issue in the damping ring design with a wiggler, composed of a series of dipole magnets of alternating polarity. In Refs. [34-40], the wiggler radiation field is calculated and the impact on the single-bunch multiturn beam instability in a damping ring has been studied [39].

However, the aforementioned MBI studies usually make the coasting beam approximation, i.e., the modulation wavelength (or the coherent radiation wavelength) is short compared with the full single-bunch duration and the modulation depth is considered as a small perturbation to the smooth background. In this paper, we will investigate the impact of undulator coherent radiation on the dynamics of a microbunch train, where the individual microbunch spacing is close to the undulator resonant wavelength. Since the unperturbed distribution is an array of microbunches, there is a distinct lack of smooth background in this situation. Due to the periodic arrangements of dipole magnets generating an alternating static magnetic field, the radiation spectrum would peak around the fundamental resonant frequency (and its integer multiples or harmonics). Near the resonant peaks, the corresponding radiation in time domain is characteristic of a relatively long-range field, for such a close spacing microbunch train. Note here that, based on conventional rf systems, the typical bunch spacing can be centimeter (or a few tens of $\mathrm{mm}$ ), and thus the aforementioned coherent-radiation-induced multibunch mechanism does not play a role in conventional storage rings. The microbunch spacing of our current interest can be about four to six orders of magnitude shorter than those based on conventional rf systems.

It has been well known that the linear accelerator (linac) driven single-pass free-electron laser (FEL) facilities typically feature extremely high-peak brightness as an electron beam is highly microbunched during the FEL process, but has a low repetition rate. Although the repetition rate can be increased by introduction of superconducting rf technology, it can be up to a few GHz [41,42]. In contrast, the conventional storage ring-based synchrotron light sources have a high repetition rate, but bear relatively low brilliance because the individual electron beams in the storage rings generally radiate incoherently. This is the motivation of the so-called steady-state microbunching (SSMB) mechanism [41-48] and subsequent investigations of the relevant collective instabilities in this new parameter regime. The SSMB is proposed to establish a circulating beam in a dedicated storage ring such that it has a phase-locked microbunch train turn after turn. At the location of the socalled radiator, the microbunch train generates coherent radiation at a high-repetition rate or close to the continuouswave mode. This underlying concept of SSMB takes 
advantage of both high-repetition rate and the resonant bunch spacing with the laser and undulator resonant wavelength. The SSMB mechanism is similar to that of beam bunching in a conventional rf system. The rf system bunches the beam with a bunch spacing equal to the microwave wavelength, and the bunching is established as a steady state on a turn-by-turn basis. The rf system also provides a longitudinal focusing so that the electron bunch has a length shorter than the rf wavelength. In SSMB, the rf system is replaced by the laser-modulator system, which consists of an infrared (IR) seed laser and an on-resonance undulator. The SSMB beam thus forms a microbunch train with bunch spacing close to the resonant wavelength. Depending on the applications, e.g., higher harmonic generation, the laser-modulator could provide longitudinal strong focusing, so that the equilibrium microbunch length much shorter than the resonant wavelength $[41,42]$.

Overall, the contributions of this work may be summarized as follows. First, we formulate the problem of the coherent-radiation-induced multibunch BBU dynamics in an undulator in presence of a steady state microbunch train based on the macroparticle model. Since the radiation field copropagates with the microbunch train at slightly different speeds, the slippage (or retardation) between the radiation and the electron beam must be properly included to obtain the physically accurate results. Second, we find that such tail-head overtaking radiation fields does perturb and under certain circumstances meanwhile provide extra focusing to the leading microbunches. Third, our developed macroparticle model is applied to two design examples, together with discussions on some physical consequences and parametric dependencies of the multibunch BBU dynamics. For the specific scenario considered in this paper, we find that when the microbunch spacing is close to the fundamental resonant wavelength, the coherent radiation can provide extra longitudinal focusing for the individual microbunches, leading to more stable multibunch oscillations.

This paper is organized as follows. In Sec. II, the undulator radiation spectrum is first briefly introduced and employed to calculate coherent radiation wake field in time domain via inverse Fourier transform. Having clarified the electron-laser-modulator system, we construct the macroparticle equations of motion and obtain a set of coupled differential equations with inclusion of the radiation slippage constraint in Sec. III A. The analytical solutions for the first few macroparticles are then obtained in Sec. III B, serving as a comparison or benchmarking with numerical solutions in Sec. IIIC. In Sec. III D, we introduce the bunching factor for the microbunch train as a convenient measure of how well the microbunch train can be preserved throughout the undulator. The choice of radiation harmonic numbers is also discussed. In Sec. IV, we apply the developed macroparticle model to the two SSMB design examples with particular emphasis on the multibunch dynamics in the modulator. Finally, we summarize and conclude the important results, outline possible future work, and discuss similarity and difference to FEL sideband instability in Sec. V.

\section{FIELD DYNAMICS}

\section{A. Radiation spectrum}

The calculation of single-electron undulator radiation has been well established in the literature. Thus in this subsection, we summarize the main results without detailed derivations. The corresponding coherent radiation field is then obtained by inverse Fourier transformation in the next subsection. The derivation of undulator radiation spectrum starts from the general formula of the angular-spectral radiation energy distribution for a prescribed electron trajectory [49]. For the electron executing wiggling motion, the angular spectral distribution can be further simplified, although the derivation is a bit tedious. Although the derivations are skipped, the interested reader is referred to Refs. [34-36,40,50,51] for more details including the discussion of the associated radiation properties. Given the period $\lambda_{w}$ and the total length $L_{w}$ of a planar undulator and the (fundamental) resonant electron energy $\gamma$, the angular spectral energy distribution for the $H$ th harmonic can be written as [40]

$$
\frac{\mathrm{d}^{2} W_{H}}{\mathrm{~d} \Omega \mathrm{d} \omega}=\frac{2 e^{2} \gamma^{2}}{\pi \epsilon_{0} c} G(\theta, \phi) F(\epsilon),
$$

where $W_{H}$ is the radiation energy-associated with the $H$ th harmonic, $\omega$ is the angular frequency, $\Omega$ is the solid angle with $\phi$ the azimuthal angle in the transverse plane, and $\theta$ is the polar angle, directed along the beam path $s$. The constants $c, \epsilon_{0}$, and $e$ are the speed of light, the vacuum permittivity, and the electron charge, respectively. The form factor $F$ is defined as

$$
F(\epsilon)=\left(\frac{\sin \pi N_{w} \epsilon}{\pi \epsilon}\right)^{2}
$$

with $\quad \epsilon=\frac{\omega}{\omega_{r}}-H=\frac{\omega}{2 c k_{w} \gamma^{2}}\left(1+\frac{K^{2}}{2}+\gamma^{2} \theta^{2}\right)-H, \quad k_{w}=2 \pi / \lambda_{w}$, $K \approx 0.934 B_{0}[$ Tesla $] \lambda_{w}[\mathrm{~cm}]$ being the dimensionless undulator parameter, $B_{0}$ is the peak undulator magnetic field, and $N_{w}=L_{w} / \lambda_{w}$ is the total number of undulator periods. The form factor $F(\epsilon)$ peaks at $\epsilon=0$ with peak value $N_{w}^{2}$ and spectral width $\approx 1 / N_{w}$. The resonance condition can be written as

$$
\lambda=\frac{\lambda_{w}}{2 \gamma^{2}}\left(1+\frac{K^{2}}{2}+\gamma^{2} \theta^{2}\right),
$$

which for the case with $\theta=0$ becomes the on axis resonance condition $\lambda=\lambda_{r}$. In Eq. (1), the angular dependence of $G$ may be related to the undulator radiation 
polarizations and can be written as the sum $G(\theta, \phi)=$ $G_{\sigma}(\theta, \phi)+G_{\pi}(\theta, \phi)$ with the horizontal contribution

$$
G_{\sigma}(\theta, \phi)=\frac{1}{2}\left[\frac{H\left(K \mathcal{D}_{1}+\frac{\gamma \theta}{\sqrt{2}} \mathcal{D}_{2} \cos \phi\right)}{1+\frac{K^{2}}{2}+\gamma^{2} \theta^{2}}\right]^{2},
$$

and the vertical contribution

$$
G_{\pi}(\theta, \phi)=\frac{1}{2}\left(\frac{H \gamma \theta \mathcal{D}_{2} \sin \phi}{1+\frac{K^{2}}{2}+\gamma^{2} \theta^{2}}\right)^{2},
$$

in which

$$
\mathcal{D}_{1}=-\frac{1}{2} \sum_{m=-\infty}^{\infty} J_{H+2 m-1}(H \alpha)\left[J_{m}(H \zeta)+J_{m-1}(H \zeta)\right]
$$

and

$$
\mathcal{D}_{2}=\sum_{m=-\infty}^{\infty} J_{H+2 m}(H \alpha) J_{m}(H \zeta)
$$

with $\alpha=\frac{2 K \gamma \theta \cos \phi}{1+\frac{K^{2}}{2}+\gamma^{2} \theta^{2}}$ and $\zeta=\frac{K^{2} / 4}{1+\frac{K^{2}}{2}+\gamma^{2} \theta^{2}}$. Here $J_{m}$ is the $m$ th order Bessel function of the first kind. Although not of our primary interest, the primary contribution comes from the sigma polarization. In practical situations, a number of radiation harmonics will be included altogether, i.e.,

$$
\frac{\mathrm{d}^{2} W}{\mathrm{~d} \Omega \mathrm{d} \omega}=\sum_{H=1}^{H_{\max }} \frac{\mathrm{d}^{2} W_{H}}{\mathrm{~d} \Omega \mathrm{d} \omega} .
$$

\section{B. Undulator radiation impedance and wake function}

In this subsection, the radiation impedance and wake function are built to accommodate the subsequent beam dynamics studies. The frequency spectrum can be obtained by integrating $\frac{\mathrm{d}^{2} W}{\mathrm{~d} \Omega \mathrm{d} \omega}$ over the solid angle $\Omega$. More specifically, we integrate the angular spectral distribution over $\phi$ and use Eq. (3) to relate the angle $\theta$ to the frequency $\omega$. The resultant expression for the $H$ th harmonic can be written as [40]

$$
\frac{\mathrm{d} W_{H}}{\mathrm{~d} \omega}=\frac{2 e^{2}}{\epsilon_{0} c} \bar{G}\left(\theta_{1}\right) \frac{1+\frac{K^{2}}{2}}{\omega / \omega_{r}} N_{w},
$$

where use has been made of $\int_{0}^{\infty} F(\epsilon) \mathrm{d} \epsilon \approx N_{w}$ and $\theta_{1}=\frac{1}{\gamma} \sqrt{\left(1+\frac{K^{2}}{2}\right)\left(H \frac{\omega_{r}}{\omega}-1\right)}$. When including up to $H_{\max }$ harmonics, $\frac{\mathrm{d} W}{\mathrm{~d} \omega}=\sum_{H=1}^{H_{\max }} \frac{\mathrm{d} W_{H}}{\mathrm{~d} \omega}$. Here $\mathrm{d} W>0$ means electron energy loss; $d W<0$ electron energy gain. In Eq. (8), the $\phi$-averaged function

$$
\bar{G}(\theta)=\frac{1}{2 \pi} \int_{0}^{2 \pi} G(\theta, \phi) \mathrm{d} \phi
$$

and $\theta$ dependence can be related to $\omega$ via Eq. (3). Here, we note that these above expressions are derived for the case of a single electron. When including all relevant harmonics of our interest, the electron energy change per unit length $\Delta W$ can be related to the impedance per unit length by $[1,2]$

$$
\Delta W=\frac{1}{2 \pi} \int_{-\infty}^{\infty} e^{2}|\tilde{\lambda}(\omega)|^{2} \operatorname{Re} Z_{\|}(\omega) \mathrm{d} \omega,
$$

where $\tilde{\lambda}(\omega)$ is the Fourier transformation of the line density distribution $\rho(z)$. For a point charge, we have $|\tilde{\lambda}(\omega)|^{2}=1$ and

$$
\operatorname{Re} Z_{\|}(\omega)=\frac{\pi}{e^{2}} \frac{\mathrm{d} W}{\mathrm{~d} \omega}
$$

For convenience of subsequent discussions using Eq. (8), we write down the undulator impedance per unit length per unit wavenmber $k_{w}=2 \pi / \lambda_{w}$ as

$$
\frac{\operatorname{Re} Z_{\|}(\omega)}{L_{w} k_{w}}=Z_{0} \bar{G}\left(\theta_{1}\right)\left(1+\frac{K^{2}}{2}\right)\left(\frac{\omega}{\omega_{r}}\right)^{-1},
$$

where $Z_{0} \approx 377 \Omega$ is the free space impedance. Using the fact that the wake function is a real quantity, the radiation impedance must have the symmetry property $Z_{\|}^{*}(\omega)=Z_{\|}(-\omega)$. Thus, we can derive the corresponding coherent radiation wake function through inverse Fourier transformation $[1,2]$

$$
W_{\|}(z>0)=\frac{2 c}{\pi} \int_{0}^{\infty} \operatorname{Re} Z_{\|}(k) \cos k z \mathrm{~d} k,
$$

with $\omega=k c$. Here, the coherent radiation fields only exist in the forward region $z>0$ and a positive wake means the energy loss. Figure 1 demonstrates a typical undulator impedance spectrum and the corresponding coherent radiation wake function, where we plot the individual harmonic components and the resulting superposition of them. In Fig. 1(a), the odd harmonics have red-shifted sharp peaks as their angular distributions are concentrated around small angles with $k \approx H k_{r}$. For even harmonics they have no intensity on axis. One can see from Fig. 1(b) that the coherent radiation fields of individual harmonics resemble a damped sinusoidal wave. It is worth making one interesting observation: the undulator radiation has long been considered a short-range field. In our past experiences this was correct, because the bunch length and bunch spacing in the conventional rf-based accelerators, both in linac-based FELs and storage rings, have much longer characteristic lengths compared with the $\mu \mathrm{m}$ or sub- $\mu \mathrm{m}$ length scale demonstrated in Fig. 1. In such situation, this 

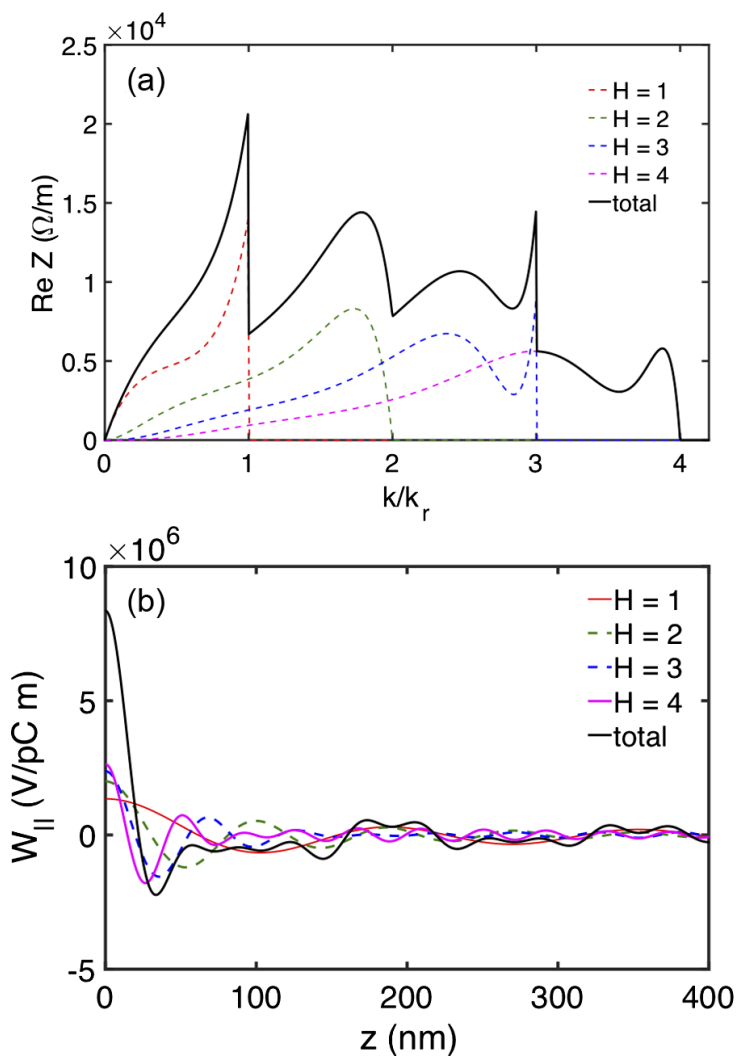

FIG. 1. The undulator radiation impedances (a) and the corresponding coherent radiation wake functions (b) for the first four individual harmonics and for the total superposition of them (black curve). Here $z>0$ indicates the bunch head or in the forward direction. The relevant parameters are $K=4.2$, $\lambda_{w}=2 \mathrm{~cm}, \gamma=\frac{400}{0.511}, k_{r} \approx 3.92 \times 10^{7} \mathrm{rad} / \mathrm{m}$.

coherent radiation field can only drive single-bunch MBI. However, as we will discuss in Sec. III, when a microbunch train is formed with bunch spacing close to the undulator resonant wavelength, such coherent radiation is no longer a short-range field and will perturb the microbunches near ahead. Before ending this section, we should make a comment on the physical meaning of the obtained radiation impedance and coherent radiation wake function. In our situation, the undulator provides the magnetic fields, and the energy exchange between the wiggling electron and the radiation field in the undulator occurs in the transverse dimension, similar to the FEL process. Therefore, the longitudinal impedance and wake function should only be considered as an equivalent form, slightly different from the conventional situation where an energy exchange happens in the longitudinal direction.

\section{ELECTRON DYNAMICS: MACROPARTICLE MODEL}

Having obtained the undulator radiation wake function, in this section, we will construct the equations of motion for the situation when a microbunch train traverses an

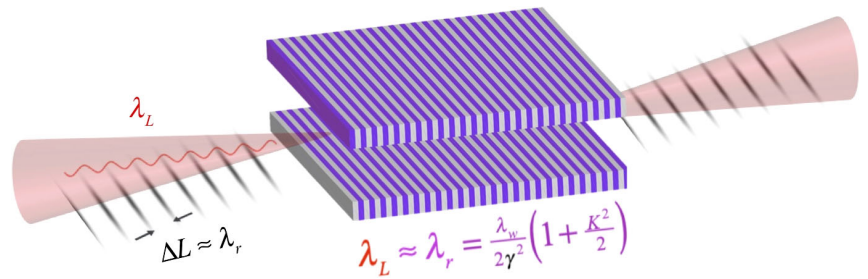

FIG. 2. Schematic layout of the modulator system. The system consists of an undulator, a microbunch train and the external seed laser. The external laser modulates the beam and provides longitudinal focusing to the individual microbunches. The undulator is designed to match the resonance condition with the external laser and the bunch spacing is set close to the undulator resonant wavelength.

undulator. Before proceeding, let us clarify the physical situation of our interest, as shown in Fig. 2. The system consists of an undulator and an electron beam with an external, copropagating seed laser. The external laser, playing a role similar to a conventional rf cavity, modulates and provides longitudinal focusing to the individual microbunches. The undulator is designed to match the resonance condition [Eq. (3)] with the external laser. The electron beam is well modulated in the form of a microbunch train, with microbunch spacing intentionally set close to the undulator resonant wavelength $\lambda_{r}$ (see Fig. 2). The undulator length is assumed a few meters to avoid FEL heating effect. Thus this system is sometimes called the modulator, in contrast to the radiator. Although we have mentioned the multipass recirculation in a dedicated SSMB storage ring, for simplicity here and in what follows we only consider the single-pass multibunch dynamics in the undulator.

\section{A. General formalism with slippage effect}

To describe the multibunch dynamics, we employ the macroparticle model, i.e., we use each macroparticle to represent an individual microbunch. The internal structure and its phase space distribution of the individual microbunches cannot be reflected in the macroparticle model, thus only serving as a simplified description. The $n$th macroparticle of a microbunch train is quantified by $z_{n}$ the longitudinal local coordinate of the bunch centroid with $z_{n}>0$ for the bunch head and $\delta_{n}=\frac{\gamma-\gamma_{0}}{\gamma_{0}}$ the relative energy offset with respect to the reference energy. Here $n$ runs from 0 to $N_{b}-1$ for the total number of $N_{b}$ microbunches. For the $n$th macroparticle, we have

$$
\frac{\mathrm{d} z_{n}}{\mathrm{~d} s}=-\eta \delta_{n}
$$

with the momentum slippage factor $\eta=-\frac{1+\frac{K^{2}}{2}}{\gamma^{2}}$. Assuming no coherent radiation field, the presence of the external seed laser and the undulator magnetic field make 
the individual microbunches execute synchrotron oscillations, thus

$$
\left(\frac{\mathrm{d} \delta_{n}}{\mathrm{~d} s}\right)_{0}=\frac{\omega_{s 0}^{2}}{\eta c^{2}} z_{n}
$$

where the subscript 0 indicates absence of collective effect. When including the overtaking radiation field, the energy change for the $n$th macroparticle can be written with the subscript 1 as

$$
\left(\frac{\mathrm{d} \delta_{n}}{\mathrm{~d} s}\right)_{1}=-\frac{4 \pi \epsilon_{0} r_{e}}{\gamma} \sum_{j=0}^{N_{b}-1} N_{j}^{\mathrm{part}} W_{\|}\left(z_{n}-z_{j}-L_{n, j}\right),
$$

where $r_{e}$ is the classical radius of the electron and $N_{j}^{\text {part }}$ is the number of electrons in the $j$ th macroparticle. Here, $L_{n, j}$ in the wake function is the longitudinal distance between the $n$th (test) macroparticle and the $j$ th (source) macroparticle, $L_{n, j}<0$ when $j<n$. The summation over $\left(N_{b}-1\right)$ is extended from $(n-1)$ by taking advantage of the fact that $W_{\|}(z<0)=0$. Here, the arguments in the wake function are the relative distances between different microbunches evaluated at the present time $s$. It is worth pointing out that the arguments in the wake function should refer to those at retarded times in the restrict sense, considering the radiation retardation effect. This replacement is largely valid if the longitudinal synchrotron motion is not evident and/or the undulator length is not that long.

Combining Eqs. (14)-(16), for the case of equal bunch spacing $L_{n, j}=-(n-j) \Delta L$, the macroparticle equation of motion for the $n$th microbunch can be written as the second-order differential equation

$$
\begin{aligned}
\frac{\mathrm{d}^{2} z_{n}}{\mathrm{~d} s^{2}}+\frac{\omega_{s 0}^{2}}{c^{2}} z_{n} & =\frac{4 \pi \epsilon_{0} r_{e} \eta}{\gamma} \sum_{j=0}^{N_{b}-1} N_{j}^{\mathrm{part}} W_{\|}\left[z_{n}-z_{j}+(n-j) \Delta L\right] \\
& \equiv \sum_{j=0}^{N_{b}-1} \mathcal{W}_{n, j},
\end{aligned}
$$

where the shorthand notation $\mathcal{W}_{n, j}=\frac{4 \pi \epsilon_{0} r_{e} \eta}{\gamma} N_{j}^{\mathrm{part}} W_{\|}\left[z_{n}-\right.$ $\left.z_{j}+(n-j) \Delta L\right]$ is introduced. When the bunch spacing is much longer than the centroid offsets $z_{n}$ of the individual microbunches, we may Taylor expand the rhs

$$
\begin{aligned}
& W_{\|}\left[z_{n}-z_{j}+(n-j) \Delta L\right] \\
& \quad \approx W_{\|}[(n-j) \Delta L]+\left.\left(z_{n}-z_{j}\right) W_{\|}^{\prime}(z)\right|_{z=(n-j) \Delta L},
\end{aligned}
$$

where the first term on RHS is a static parasitic loss term independent of the dynamics and sometimes ignored. Then, Eq. (17) becomes

$$
\begin{aligned}
\frac{\mathrm{d}^{2} z_{n}}{\mathrm{~d} s^{2}}+\frac{\omega_{s 0}^{2}}{c^{2}} z_{n} & \left.\approx \frac{4 \pi \epsilon_{0} r_{e} \eta}{\gamma} \sum_{j=0}^{N_{b}-1} N_{j}^{\mathrm{part}}\left(z_{n}-z_{j}\right) W_{\|}^{\prime}(z)\right|_{z=(n-j) \Delta L} \\
& \equiv \sum_{j=0}^{N_{b}-1}\left(z_{n}-z_{j}\right) \mathcal{W}_{n, j}^{\prime}\left(-L_{n, j}\right) .
\end{aligned}
$$

Here, we have introduced a similar shorthand notation $\mathcal{W}_{n, j}^{\prime}$. On the rhs, the quantity $\left(z_{n}-z_{j}\right)$ resembles the derivative $\mathrm{d} z / \mathrm{d} s$, suggesting a possible instability [1]. In Eq. (19), $W_{\|}^{\prime}$ can be evaluated by differentiating Eq. (13) with respect to $z$

$$
W_{\|}^{\prime}(z>0)=-\frac{2 c}{\pi} \int_{0}^{\infty} k \operatorname{Re} Z_{\|}(k) \sin k z \mathrm{~d} k .
$$

Here, we note that Eqs. (17) and (19) are not our final forms for the macroparticle equations of motion. Since the copropagating radiation fields traveling at the speed of light $c$ will slip on the electron beam (with $v_{z}<c$ ) over the radiation wavelength $\lambda_{r}$ in each undulator period $\lambda_{w}$ [see Eq. (3)], this constitutes the slippage constraint that, for two neighboring microbunches with bunch spacing $\Delta L$, for the trailing microbunch to catch up the leading one, $\Delta L \leq N_{k} \lambda_{r}$ or $N_{k} \geq \Delta L / \lambda_{r}$ must be fulfilled. Here $N_{k}=$ $-L_{k, k-1} / \lambda_{r}$ is the distance between the $k$ th and $(k-1)$ th microbunches in terms of the resonant wavelength. The slippage condition between arbitrary two microbunches can be found by the similar arguments. Therefore for the $n$th microbunch, we have the modified equation of motion

$\frac{\mathrm{d}^{2} z_{n}}{\mathrm{~d} s^{2}}+k_{s}^{2} z_{n}=-\sum_{j=0}^{n-1} \mathcal{W}_{n, j}^{\prime} \Theta\left(s-\lambda_{w} \sum_{k=j}^{n-1} N_{k+1}\right) z_{j}$,

where $\Theta$ is the Heaviside unit step function and the rhs of Eq. (19), characterizing the PWD term, is combined in the perturbed synchrotron oscillation wavenumber $k_{s}$

$$
k_{s}^{2}=\frac{\omega_{s 0}^{2}}{c^{2}}-\sum_{j=0}^{n-1} \mathcal{W}_{n, j}^{\prime} \Theta\left(s-\lambda_{w} \sum_{k=j}^{n-1} N_{k+1}\right),
$$

where $N_{k+1}=-\frac{L_{k+1, k}}{\lambda_{r}}$. Note that when writing down Eq. (21), we have assumed that displacements of the microbunch centroids are small compared with the microbunch spacing. This is valid in most situations of the storage ring or linac-based accelerators. For the scenario considered in this paper, however, the contribution of coherent radiation fields to $k_{s}$ is not necessarily a small perturbation. In other words, the Eq. (21), resulting from Eq. (18), may no longer be accurate; eventually we shall come back to Eq. (17) along with slippage constraint.

Although we are primarily interested in the single-pass multibunch dynamics in an undulator, the synchrotron 
radiation damping and diffusion can be added on rhs of Eq. (17) for multipass studies if considering a storage ring configuration. It deserves here to notice a difference of Eq. (21) from the equation that describes the conventional longitudinal BBU instability $[1,2,6]$. In the conventional $\mathrm{BBU}$, the wake or impedance is localized, e.g., in a cavitylike structure, and is left by the preceding beam bunches. The following bunches would experience the wake or impedance. This process involves the causality condition (wake only exists for $z<0$ ) and the slippage or retardation effect is absent. Equation (18) is valid in most situations and the unit step function is not necessary in Eq. (21). Since the radiation field is not localized but copropagating with the electron beam, the slippage between the radiation and the electron beam is properly counted to obtain the physically accurate results. The slippage effect becomes important when the length of the undulator is short and the microbunch train is long. Furthermore, Eq. (18) is held valid only when $(n-j) \Delta L \gg z_{n}-z_{j}$. In the situation of $\Delta L \approx \lambda_{r}$, the coherent radiation field can be a large perturbation to the leading microbunches, resulting in the bunch centroid offset $z_{n}$ comparable to the $\lambda_{r}$.

\section{B. Analytical solutions for a few trailing macroparticles}

After the first-order Taylor expansion [Eq. (18)], Eq. (21) is a linearized differential equation. The analytical solution for this equation can be obtained. Here, we only find the solutions for the first two macroparticles, discuss the features associated with Eq. (21) and will use them as a benchmark against numerical solutions in the next subsection. The zeroth macroparticle is expected to execute a free synchrotron oscillation,

$$
z_{0}=\hat{z}_{0} \cos \left(k_{s 0} s\right)
$$

for $0<s<L_{w}$, where $\hat{z}_{0}$ is the initial offset. The longitudinal focusing $k_{s 0}$ comes from the external laser only.

Before the radiation fields overtake, the first macroparticle executes a synchrotron oscillation, similar to the zeroth macroparticle

$$
z_{1}=\hat{z}_{1} \cos \left(k_{s 0} s\right)
$$

for $0<s<N_{1} \lambda_{w}$, with the initial offset $\hat{z}_{1}$. For $s>N_{1} \lambda_{w}$, the radiation fields catch up. The macroparticle equation of motion is now appended to the driving term

$$
\frac{\mathrm{d}^{2} z_{1}}{\mathrm{~d} s^{2}}+\left(\frac{\omega_{s 0}^{2}}{c^{2}}-\mathcal{W}_{1,0}^{\prime}\right) z_{1}=-\mathcal{W}_{1,0}^{\prime} z_{0},
$$

for $N_{1} \lambda_{w}<s<L_{w}$. To find the solution in a convenient way, let us define the coordinate $s_{*}=s-N_{1} \lambda_{w}$. The initial conditions for Eq. (25) can be written as

$$
\begin{aligned}
& z_{1}\left(s=N_{1} \lambda_{w}\right)=\hat{z}_{1} \cos \left(k_{s 0} N_{1} \lambda_{w}\right) \equiv z_{1}\left(s_{*}=0\right), \\
& z_{1}^{\prime}\left(s=N_{1} \lambda_{w}\right)=-k_{s 0} \hat{z}_{1} \sin \left(k_{s 0} N_{1} \lambda_{w}\right) \equiv z_{1}^{\prime}\left(s_{*}=0\right) .
\end{aligned}
$$

It is straightforward to obtain the general solution

$$
\begin{aligned}
z_{1}\left(s_{*}\right)= & z_{1}\left(s_{*}=0\right) \cos \left(k_{s} s_{*}\right)+\frac{\mathcal{W}_{1,0}^{\prime} \hat{z}_{0}}{k_{s}^{2}-k_{s 0}^{2}} \cos \varphi \cos \left(k_{s} s_{*}\right) \\
& +z_{1}^{\prime}\left(s_{*}=0\right) \frac{\sin \left(k_{s} s_{*}\right)}{k_{s}}-\frac{k_{s 0}}{k_{s}} \frac{\mathcal{W}_{1,0}^{\prime} \hat{z}_{0}}{k_{s}^{2}-k_{s 0}^{2}} \sin \varphi \sin \left(k_{s} s_{*}\right) \\
& -\frac{\mathcal{W}_{1,0}^{\prime} \hat{z}_{0}}{k_{s}^{2}-k_{s 0}^{2}} \cos \left(k_{s 0} s_{*}+\varphi\right)
\end{aligned}
$$

where $\mathcal{W}_{1,0}^{\prime}=\mathcal{W}_{1,0}^{\prime}\left(-L_{1,0}\right)$ and $\varphi=k_{s 0} N_{1} \lambda_{w}$. When the radiation field is a small perturbation, we have $k_{s} \approx k_{s 0}$ and Eq. (26) can be further simplified to be

$$
\begin{gathered}
z_{1}\left(s_{*}\right) \approx z_{1}\left(s_{*}=0\right) \cos \left(k_{s 0} s_{*}\right)+z_{1}^{\prime}\left(s_{*}=0\right) \frac{\sin \left(k_{s 0} s_{*}\right)}{k_{s 0}} \\
-s_{*} \frac{\mathcal{W}_{1,0}^{\prime} \hat{z}_{0}}{2 k_{s 0}} \sin \left(k_{s 0} s_{*}+\varphi\right),
\end{gathered}
$$

where the first and second terms on rhs originate from a free synchrotron oscillation. The third term, grows linearly with $s$, is a resonant driving term. One can show that for the $j$ th macroparticle, the resonant driving terms is a sum of $s$-polynomials up to the order $j$. In the next subsection, we will compare the analytical solutions obtained with the numerical solutions [Eqs. (17) and (21)] and discuss some features associated with the derived macroparticle model.

\section{Comparison with numerical solutions}

Before proceeding, it is worth remembering the three situations relevant to our studies. The first situation involves the slippage constraint; this condition is applied by introducing an $s$-dependent unit step function for the $n$th macroparticle associated with the trailing $j$ th macroparticles, $\Theta\left(s-\lambda_{w} \sum_{k=j}^{n-1} N_{k+1}\right)$. The second situation is related to linearization of the undulator radiation wake function, cf. Eqs. (17) and (19). For convenience of the following discussion, Eq. (17) is referred to the nonlinear case, and Eq. (19) to the linear case. The third concerned situation is inclusion of the potential-well distortion (PWD) effect [Eqs. (21) and (22)]. The nonlinear case, Eq. (17), intrinsically includes the potential-well term. For the linear case [Eq. (19) or (21)], in the absence of PWD $k_{s}=k_{s 0}$ is assumed. Here, the longitudinal focusing is merely provided by external laser and will be estimated in Sec. IV. In the following discussion of this subsection, we will explore the above three situations. For simplicity, we assume only the fundamental harmonic is included in the undulator radiation field in this subsection, i.e., 

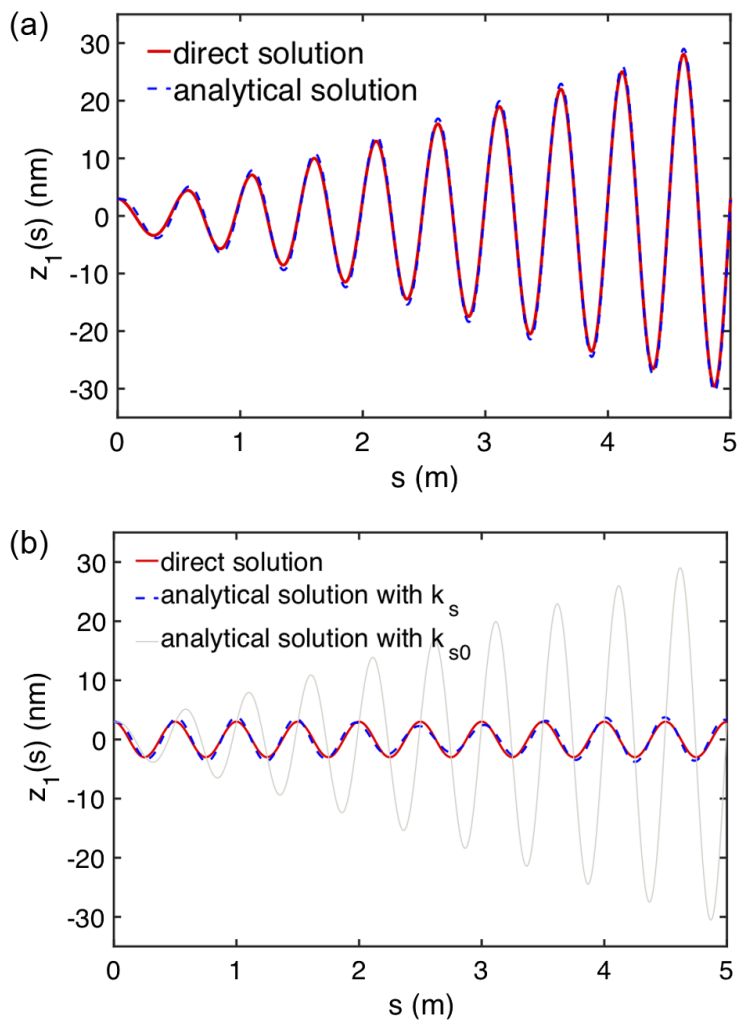

FIG. 3. Solutions to the first macroparticle. (a) Without potential-well effect, the red curve from Eq. (21) with $k_{s}=k_{s 0}$ and the blue dashed curve from Eq. (27). (b) With potential-well effect, the red curve from the same Eq. (21) with $k_{s}$ given by Eq. (22) and the blue dashed curve from Eq. (26). For reference purposes, the gray curve in (b) is identical to the blue dashed curve in (a). The slippage effect is included in all the cases. Here $\hat{z}_{1}(0)=3 \mathrm{~nm}, K=4.2, \lambda_{w}=2 \mathrm{~cm}$, $k_{s 0}=4 \pi, k_{s} \approx 4.6 \pi, N_{\mathrm{pb}}=1.875 \times 10^{7}, Q=3 \mathrm{pC}, \gamma \approx 783$, $\Delta L=\frac{2 \pi}{k_{r}} \approx 0.16 \mu \mathrm{m}, H_{\max }=1$.

$H_{\max }=1$. We will discuss the choice of the radiation harmonics $H_{\max }$ in the next subsection.

Figure 3 shows the longitudinal bunch centroid oscillation of the penultimate $(n=1)$ microbunch along $s$. The microbunch train is composed of four microbunches with the identical initial conditions. The initial offsets $\hat{z}_{n}(0)=$ $3 \mathrm{~nm}$ for $n=0 \sim 3, K=4.2, \lambda_{w}=2 \mathrm{~cm}$, the number of electrons per microbunch $N_{\mathrm{pb}}=1.875 \times 10^{7}$, corresponding to $Q=3 \mathrm{pC}$, and the reference electron energy $400 \mathrm{MeV}$. The choice of the initial numerical small offsets can be somewhat arbitrary simply to avoid the trivial case with $\hat{z}_{n}(0)=0$ [see Eq. (21)]. From the resonance condition, the undulator resonant wavelength, hence the bunch spacing, is about $0.16 \mu \mathrm{m}$. Figure 3(a) demonstrates the situation when the macroparticle is resonantly driven by the undulator radiation field, while executing the unperturbed$k_{s 0}$ synchrotron oscillation. The analytical solution Eq. (27) and the direct numerical solution from Eq. (21) with $k_{s}=k_{s 0}$ are in perfect agreement. It is straightforward to solve the system of differential equations [Eq. (21)] using the numerical standard algorithms. Here, the fourth-order Runge-Kutta integration is employed. In Eq. (22), we set $k_{s 0}=4 \pi$; this number is only for the demonstration purpose and can be exaggerated by one order of magnitude than in practical situations. The potential-well term is included in Fig. 3(b), where we find the oscillation is stabilized; see the gray curve in the background of the figure for the case without PWD. The analytical solution, based on Eq. (26), is still in good agreement with the direct numerical solution from Eq. (21) with $k_{s} \approx 4.6 \pi$.

From Eq. (27) in Sec. III B, we comment that for the $j$ th macroparticle the resonant driving terms is a sum of $s$-polynomials up to the order $j$, i.e., $z_{j}(s) \propto \sum_{p=0}^{j} s^{p}$, $j=0,1,2, \ldots, N_{b}-1$. When the undulator is sufficiently long, the oscillation envelope is proportional to $s^{j}$. This statement however may not be helpful because the strong assumption of $k_{s} \approx k_{s 0}$ is made [see also Eq. (18)]. We will see in Fig. 4 that Eq. (21) would break down when an undulator is long or the microbunch charge is high. To further appreciate the important role of the potential-well term [Eq. (22) or intrinsically included in Eq. (17)], Fig. 4 shows the oscillation from the most leading microbunch ( $n=3$ for a microbunch train of $N_{b}=4$ ) in three different cases: (i) the linear model without potential-well term, (ii) the linear model with potential-well term, and (iii) the nonlinear model. The slippage effect is included in the three cases. It deserves to make a few comments here. The oscillation amplitude in the case (i) is larger than the other two cases when $s>3 \mathrm{~m}$. This asymptotic behavior, a cubic in $s$, is due to resonant driving. When including the potential-well term, the cases (ii) and (iii) become different

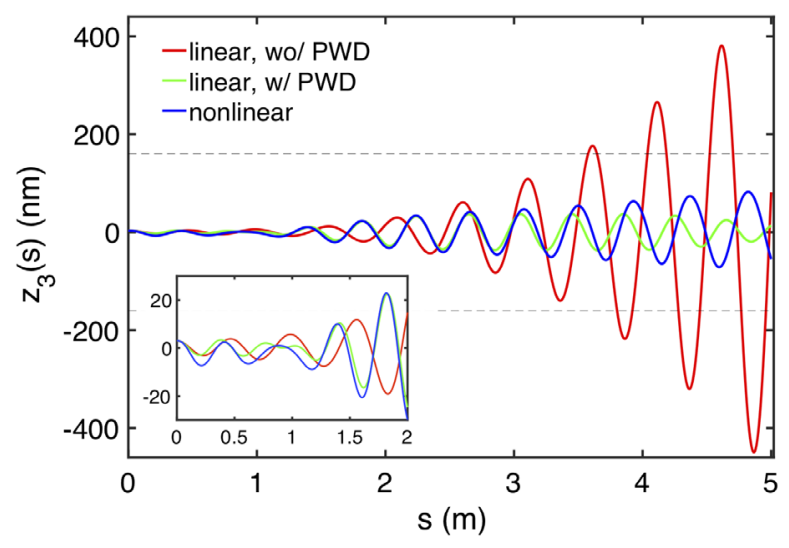

FIG. 4. Evolution of the most leading $(n=3)$ macroparticle along $s$ based on three different cases. The slippage effect is included. The red curve is obtained from Eq. (21) with $k_{s}=k_{s 0}$; the green curve from Eqs. (21) with $k_{s}$ given in Eq. (22); the blue curve from Eq. (17), where the potential-well effect is intrinsically included. The inset shows a zoom for the oscillation in $0<s<2 \mathrm{~m}$. Here the initial microbunch offsets assume $\hat{z}_{n}(0)=$ $(-1)^{n+1} \times 3 \mathrm{~nm}(n=0 \sim 3)$, and the remaining parameters are the same as those assumed in Fig. 3. 
from the case (i), as shown in Fig. 4 the green and blue curves. While this microbunch is subject to an unstable resonant driving, the potential-well term provides extra focusing and stabilization to the longitudinal oscillation. The oscillation wavenumber $k_{s}$ larger than $k_{s 0}$ can also be seen in the oscillation pattern. Further examination of the cases (ii) and (iii) [the green and blue curves] indicates that after $s>3 \mathrm{~m}$ the linear model overestimates the stabilization effect. The slight difference at $s<2 \mathrm{~m}$ between the cases (ii) and (iii), shown in the inset, indeed comes from the parasitic loss, the first term on rhs of Eq. (18). When adding it back, the difference between the two cases becomes negligible at $s<2 \mathrm{~m}$.

In Sec. III A, we have introduced the slippage or retardation effect, which will be important for a short undulator or long microbunch train. Figure 5 illustrates the most leading microbunch of a microbunch train with $N_{b}=50$ along a 2-m undulator. The input parameters are basically the same as those used in the previous discussion. It can be seen that, when the slippage constraint is included, the oscillation pattern (red curve) at the beginning distance (say $s<0.5 \mathrm{~m}$ ) is not exactly but resembles a free synchrotron oscillation. In contrast, if neglecting the slippage condition, we would obtain an overestimated energy kick from the trailing microbunches (blue curve). Note that the two curves in Fig. 5 are based on the nonlinear model [Eq. (17)]; therefore both the potential-well term and the parasitic loss have been included.

\section{Bunching factor for microbunch train and choice of harmonics number}

As outlined at the beginning of this section, when the microbunch train is formed, it circulates in the modulator system in a dedicated storage ring. Then, we expect the microbunch train to radiate coherently in the so-called radiator system. Therefore in the modulator, it is our hope

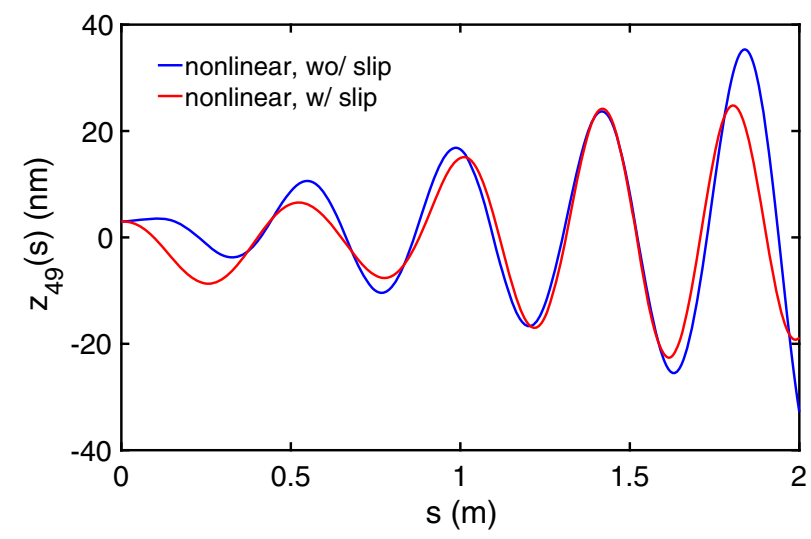

FIG. 5. Evolution of the most leading $(n=49)$ macroparticle along $s$ for a bunch train with 50 microbunches. The blue and red curves are obtained from Eq. (17) without and with the slippage condition, respectively. The parameters are the same as those used in Fig. 4. to well preserve the multibunch oscillation from the perturbation by the coherent and collective interactions with the environment. For the purpose of the analysis let us define the bunching factor for the bunch train

$$
\mathcal{B}(k) \equiv\left|\left\langle e^{i k z_{j}}\right\rangle\right|=\frac{1}{N_{b}}\left|\sum_{j=0}^{N_{b}-1} e^{i k z_{j}}\right|,
$$

where the bracket $\langle\cdots\rangle$ refers to the average over all the microbunches. This definition is not different from the familiar one used to quantify the microbunching in FEL or in a transport line for a coasting beam. For the on resonance case, $\Delta L=2 \pi / k_{r}, k_{r}=2 \gamma^{2} /\left(1+\frac{K^{2}}{2}\right), z_{j}=j \Delta L$, the bunching factor can be further simplified

$$
\mathcal{B}(k)=\frac{1}{N_{b}}\left|\sum_{j=0}^{N_{b}-1} e^{i\left(\frac{k}{k_{r}}\right) j 2 \pi}\right|=\frac{1}{N_{b}}\left|\frac{\sin \left(\frac{k}{k_{r}}\right) N_{b} \pi}{\sin \left(\frac{k}{k_{r}}\right) \pi}\right| .
$$

In absence of collective effects, i.e., the pure optics case, we have the on resonance bunching factor $\mathcal{B}\left(m k_{r}\right)=1$ with $m$ an integer. For a bunch train with $N_{b}$ microbunches, the local maximum of the bunching factor not only occurs at the integer multiples of $k_{r}$ but also at $\frac{k}{k_{r}} \approx p+$ $\frac{4 n \pm 1}{2 N_{b}}$ with $p$ the integer and $1<n<\left(\frac{N_{b}}{2}-1\right)$ with the local values $\mathcal{B}(k) \approx \frac{1}{2 n \pi \pm \frac{\pi}{2}}$.

As for the choice of the harmonic number in the undulator radiation spectrum, it depends on the design goal or/and the intrinsic beam energy spread $\sigma_{\delta}$. The equivalent momentum compaction factor for the undulator can be obtained from Eq. (3) and $|\eta|=\left|R_{56}\right| / L_{w}$, giving

$$
\left|R_{56}\right|=2 N_{w} \lambda_{r} .
$$

When traversing the undulator, the uncorrelated beam energy spread will induce longitudinal smearing in $z$, leading to $|\delta z| \approx\left|R_{56}\right| \sigma_{\delta}$, within which the radiation is not coherent. Thus, the maximum harmonic number of our interest can be determined by

$$
H_{\max } \leq 1 / 2 N_{w} \sigma_{\delta} .
$$

In the macroparticle model, the finite energy spread of an individual microbunch is not included. Therefore Eq. (31) only serves as a reference for the moment. The number of radiation harmonics can also be considered as one of the design value when the microbunch train is aimed to radiate for the high harmonic radiation.

Before ending this section, let us summarize what we have obtained. In the first two subsections, we constructed the macroparticle equations of motion and obtained the analytical solution together with the slippage constraint. By linearizing the wake function Eq. (18), we have the linear equation Eqs. (21) and (22) with the potential-well term 
included as a perturbed source to the synchrotron oscillation wavenumber $k_{s}$. The numerical solutions to the macroparticle equations of motion are benchmarked with the analytical solution for some special cases.

\section{EXAMPLES}

In this section, we will illustrate two design examples based on the longitudinal strong focusing SSMB scenario [41]. One of them is designed to produce high repetition rate, $\mathrm{kW}$ average power, coherent extreme ultraviolet (EUV) radiation source with the targeted wavelength at $13.5 \mathrm{~nm}$. The other example aims for the longer wavelength in the IR region. The relevant beam and undulator parameters for the two examples are listed in Table I. Here, we notice that the numbers in Table I are slightly modified from those in Ref. [41]. More precisely, the electron numbers per microbunch for the EUV case used in this paper is about 30 times larger than those quoted in the nominal design of the average current $1 \mathrm{~A}$ and that for the IR case is about 150 times larger [41]. In the two examples, our discussions, though with more emphasis on the first one, will be restricted in the single-pass multibunch dynamics in the undulator of the modulator system. Although the number of microbunches in the bunch train can be relatively limited in our numerical setup, the discussions are in fact generally applicable to a larger number of microbunches.

\section{A. Example 1: EUV}

In the first example, dubbed as EUV, we consider a bunch train with ten microbunches. Some relevant parameters are listed in Table I. The maximum radiation harmonic number $H_{\max }$ is set to 13 by design. For our subsequent discussions, we increase the length of the undulator from the design value $2-10 \mathrm{~m}$, where the laser voltage of the modulator is also increased from 0.51 to $2.55 \mathrm{MV}$ to ensure the same longitudinal focusing $k_{s 0}$ and the bucket height throughout the undulator. The longitudinal oscillation wavenumber $k_{s 0}$ and the half bucket height provided by the external laser in the modulator can be estimated as [52]

TABLE I. Relevant beam and undulator parameters for the two examples EUV and IR.

\begin{tabular}{lccc}
\hline \hline Name & EUV & IR & Unit \\
\hline Electron beam energy & 400 & 400 & $\mathrm{MeV}$ \\
Electron numbers per microbunch $1.2 \times 10^{5}$ & $4.38 \times 10^{7}$ & \\
Electron charges per microbunch & 0.02 & 7 & $\mathrm{pC}$ \\
Undulator length & 2.0 & 2.1 & $\mathrm{~m}$ \\
Undulator period & 2.2 & 9.6 & $\mathrm{~cm}$ \\
Resonance wavelength & 0.176 & 12.8 & $\mu \mathrm{m}$ \\
Modulator voltage & 0.51 & 0.42 & $\mathrm{MV}$ \\
Unperturbed synchrotron & 0.54 & 0.214 & $\mathrm{rad} / \mathrm{m}$ \\
$\quad$ & & & \\
\hline \hline
\end{tabular}

$$
k_{s 0} \approx \sqrt{\frac{e \mathcal{E}_{0} K[J J] k_{w}}{\gamma^{2} m c^{2}}},
$$

and

$$
\delta_{\frac{1}{2}} \approx \frac{k_{s 0}}{k_{w}}=\sqrt{\frac{e \mathcal{E}_{0} K[J J]}{\gamma^{2} m c^{2} k_{w}}},
$$

with $[J J]=J_{0}(\chi)-J_{1}(\chi), \chi=K^{2} /\left(4+2 K^{2}\right)$ the electric field amplitude $\mathcal{E}_{0} \approx 2 \gamma V_{m} / K L_{w}$ and $V_{m}$ being the modulator voltage [40]. For the numerical values given in Table I, we have $\mathcal{E}_{0} \approx 95 \mathrm{MV} / \mathrm{m}, k_{s 0} \approx 0.54 \approx \frac{2 \pi}{11.64} \mathrm{rad} / \mathrm{m}$, and $\delta_{\frac{1}{2}} \approx 1.7 \times 10^{-3}$.

Figure 6 is obtained by numerically solving Eq. (17) without making an approximation of the centroid offsets of the individual microbunches. One can see from the figure that the most leading microbunch $(n=9)$ is subject to resonant driving when $s$ increases. The resonantly driven macroparticles are also characteristic of increasing synchrotron oscillation wavenumbers. For the 9-th macroparticle, the synchrotron wavenumber ranges from 2.7 to $4.6 \mathrm{rad} / \mathrm{m}$, corresponding to the varying oscillation wavelengths from $2.3 \mathrm{~m}$ down to $1.4 \mathrm{~m}$. Note here that the bunch spacing is about $176 \mathrm{~nm}$ (see Table I), much larger than the longitudinal centroid offsets of the individual microbunches.

To further appreciate the extra focusing provided by the coherent undulator radiation fields, we use the linear model [Eq. (21)] without including the potential-well term $\left(k_{s}=k_{s 0}\right)$ as a comparison with Fig. 6. Using the same parameters as in Figs. 6 and 7 shows the severely resonant driven microbunches. The growing amplitudes are already much larger than those in Fig. 6 at $s=2 \mathrm{~m}$, where the linear model can break down. Because of the absence of

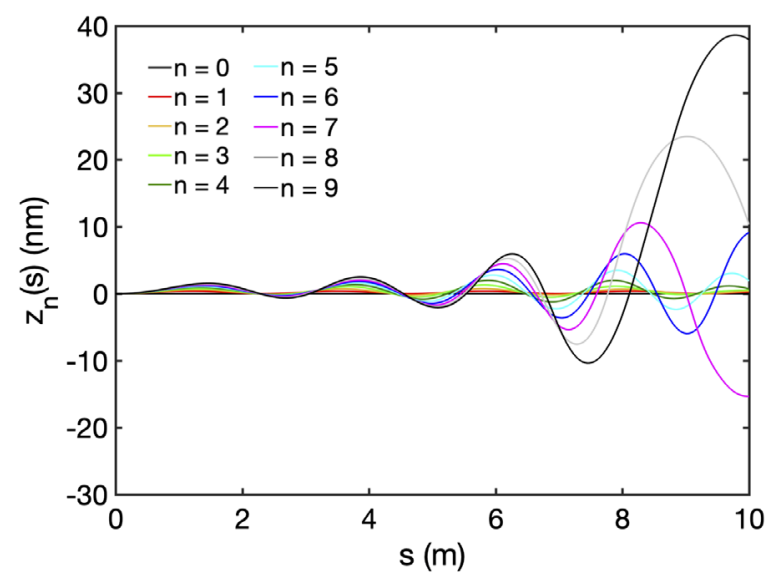

FIG. 6. Evolution of the longitudinal centroid offsets along $s$ based on Eq. (17). Here, we assume the initial offsets $\hat{z}_{n}(0)=0$ for all ten microbunches $(n=0-9)$. 


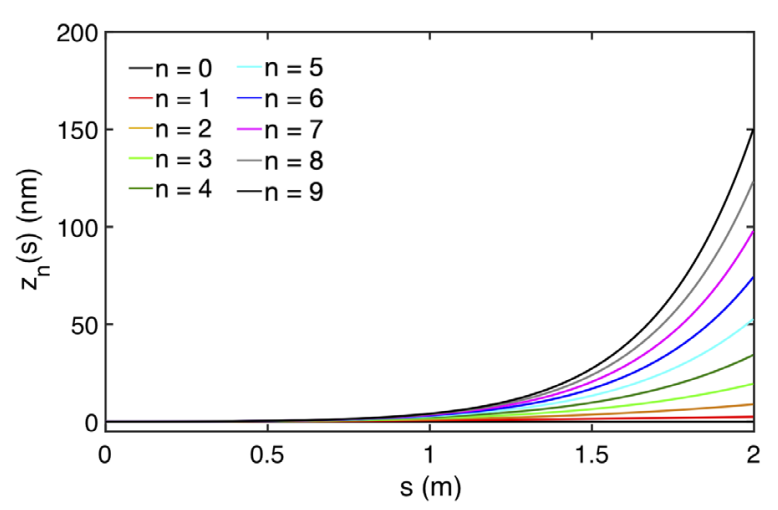

FIG. 7. Evolution of the longitudinal centroid offsets along $s$ based on Eq. (21) with $k_{s}=k_{s 0}$, i.e., without potential-well term. The initial parameters are the same as those in Fig. 6.

potential-well term, $k_{s 0}=0.516$, the microbunches do not even execute a full synchrotron oscillation at $s=2 \mathrm{~m}$.

Let us further investigate the multibunch dynamics within the framework of the macroparticle model. We can draw the macroparticle phase space portrait $\left(z_{n}, \delta_{n}=-\frac{z_{n}^{\prime}}{\eta}\right)$ to examine the dynamics of the microbunch train. Note here that the individual microbunch is represented by a single macroparticle. The evolution of such phase space portrait along $s$ may be viewed as the evolution of the microbunch centroids $\left(z_{n}, \delta_{n}\right)$ in phase space, and is distinct from the familiar phase space distribution for the individual electrons. From Eqs. (14) and (15), the macroparticles would rotate clockwise in the phase space portrait.

Figure 8 demonstrates the three situations of our interest. Figure 8(a) refers to the case at $s=2 \mathrm{~m}$ based on Table I, where the macroparticle trajectories are solved based on the nonlinear model Eq. (17) with inclusion of the slippage effect. As a comparison, Fig. 8(b) shows the case with the linear model [Eq. (21)] including the slippage but without the potential-well term. From Figs. 8(a) and 8(b) we again confirm that the potential-well effect provides extra longitudinal focusing for individual microbunches, leading to relatively stabilized synchrotron motion. As a reference, the phase space portrait at $s=10 \mathrm{~m}$ is shown in Fig. 8(c), where the four leading microbunches execute a relatively large-amplitude synchrotron oscillation. We point out that, here and in what follows, the stable phase space area enclosed by the separatrices in the figures should be a bit smaller than expected, as the separatrices are drawn only based on the pendulum Hamiltonian without taking into the coherent undulator radiation field into consideration.

As introduced in Sec. III D, for a microbunch train the bunching factor defined in Eq. (28) can be used to quantify the evolution of the microbunch train along $s$ in terms of the normalized wavenumber. For the three cases in Fig. 8, we calculate the bunching spectrum as a function of $s$ and $k / k_{r}$, where Fig. 8(b) corresponds to the right panel of Fig. 9 and Figs. 8(a) and 8(c) to the left panel of Fig. 9. It can be seen (a)

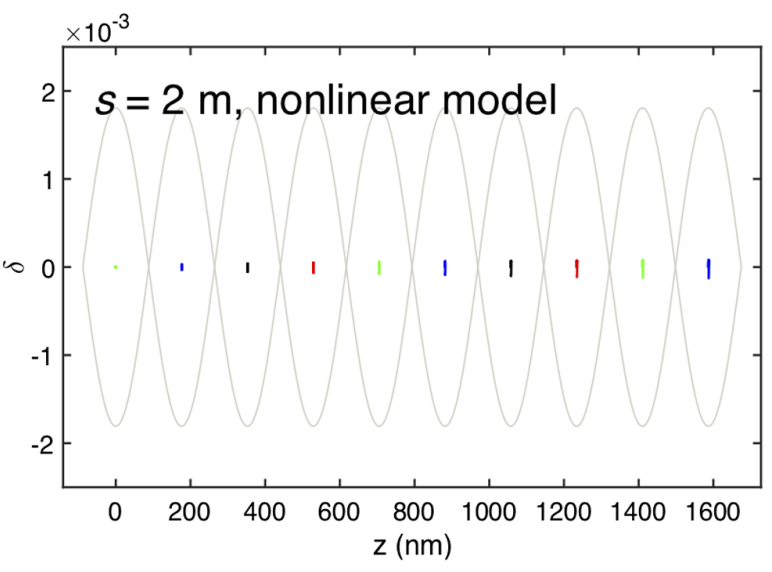

(b)

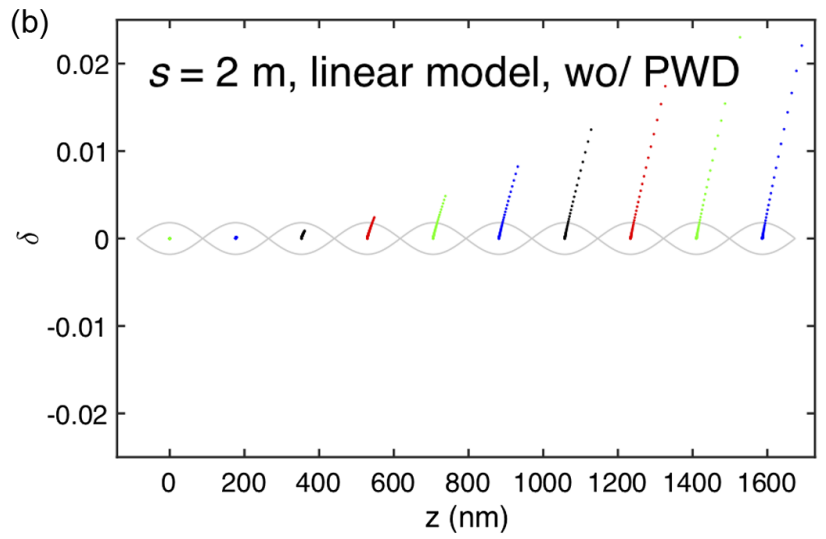

(c)

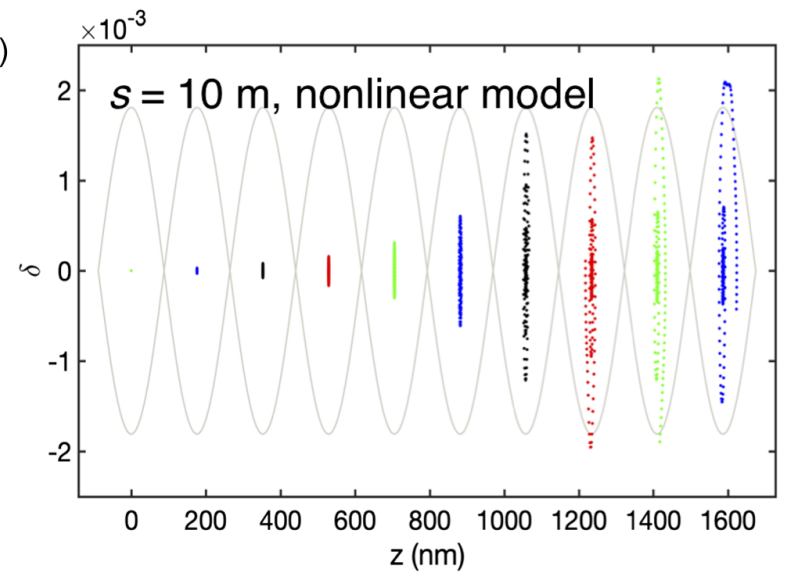

FIG. 8. Macroparticle phase space evolution for individual microbunches (a) at $s=2 \mathrm{~m}$ based on the nonlinear model [Eq. (17)]; (b) at $s=2 \mathrm{~m}$ based on the linear model [Eq. (21)] and neglecting potential-well term; (c) at $s=10 \mathrm{~m}$ based on the nonlinear model. Here $H_{\max }=13$. For (c), the modulator voltage is adjusted to retain the same laser field as that for (a).

from Figs. 9(a) and 9(c) that at $s=2 \mathrm{~m}$ the microbunch train is well preserved. After $s=6 \mathrm{~m}$, the bunching factors of higher harmonics begin to degrade. The sideband signals emerge due to large-amplitude synchrotron oscillation at $s=10 \mathrm{~m}$, also shown in Fig. 9(c). Comparing Figs. 9(c) with 9 (d), thanks to the additional longitudinal focusing 

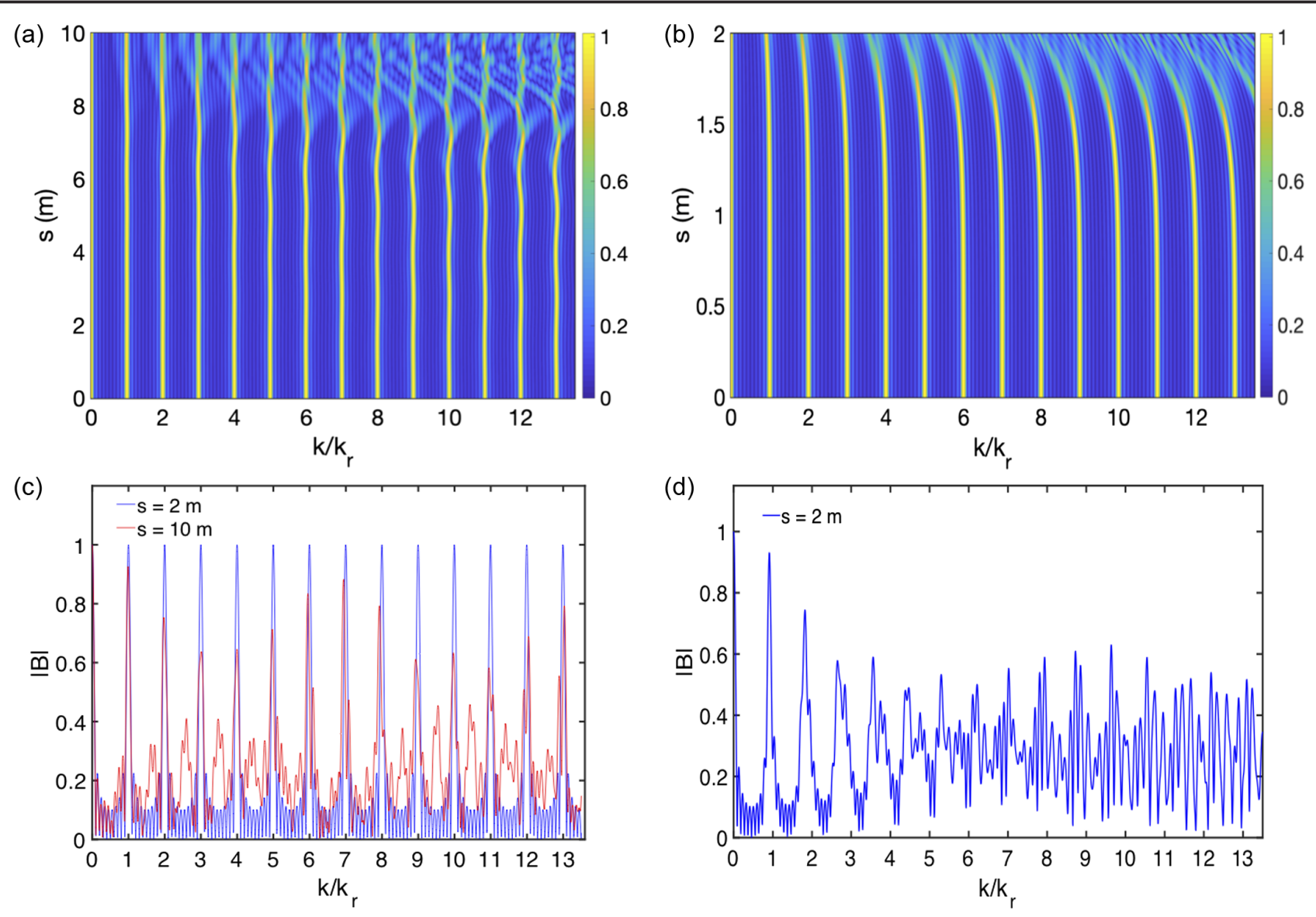

FIG. 9. Evolution of the bunching spectrum along the undulator as a function of the normalized wavenumber. (a,c) from the nonlinear model; (b,d) from the linear model without potential-well term. From (c), the sideband appears due to large-amplitude synchrotron oscillation. See the context for more discussions.

provided by the coherent radiation field, the harmonic bunching factors have been well preserved.

The dependence of the bunching factor on the microbunch charge may also be of our interest. Figure 10 gives $|\mathcal{B}|$ at $H=1, H=7$, and $H=13$ as a function of the

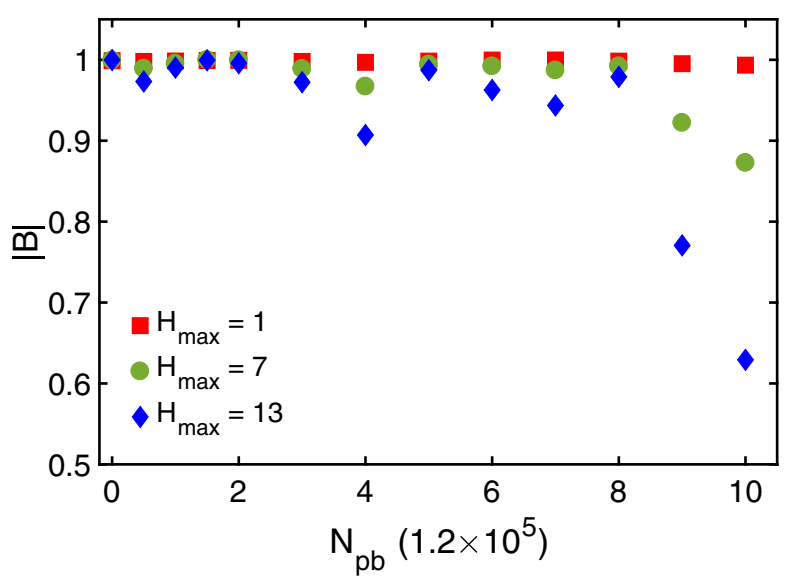

FIG. 10. Dependence of the bunching factors $|\mathcal{B}|$ on the number of electrons per microbunch for different radiation harmonics. The bunching factor is evaluated at $s=2 \mathrm{~m}$. number of electrons per microbunch at $s=2 \mathrm{~m}$. The higher microbunch charges imply more evident collective multibunch oscillations. As expected, the higher-harmonic bunching factors become more sensitive to the centroid offsets of individual microbunches, and would degrade as the microbunch intensity increases. For the case of $H_{\max }=13$, the bunching factor remains larger than 0.9 for the bunch intensity eight times the design value. Since SSMB aims for high-repetition rate and high-average radiation power, we have $P_{\text {ave }} \propto f N_{\mathrm{pb}} N_{\text {coh }}$ with $f$ the repetition rate, $N_{\mathrm{pb}}$ the number of electrons per microbunch, and $N_{\text {coh }}$ the number of electrons within one coherent wavelength [41]. Assuming the length of the individual microbunches is shorter than the undulator resonant wavelength, when the microbunch charge doubles, the output power becomes squared. We have only indeed considered the impact of coherent radiation field in the undulator and ignored other collective effects when the microbunch train leaves the modulator for the radiator.

We further investigate an interesting situation, i.e., when the microbunch charges are not uniform in the bunch train. Let us consider, starting from the $n=0$ microbunch, an increasing sequence of the individual microbunch charges, 

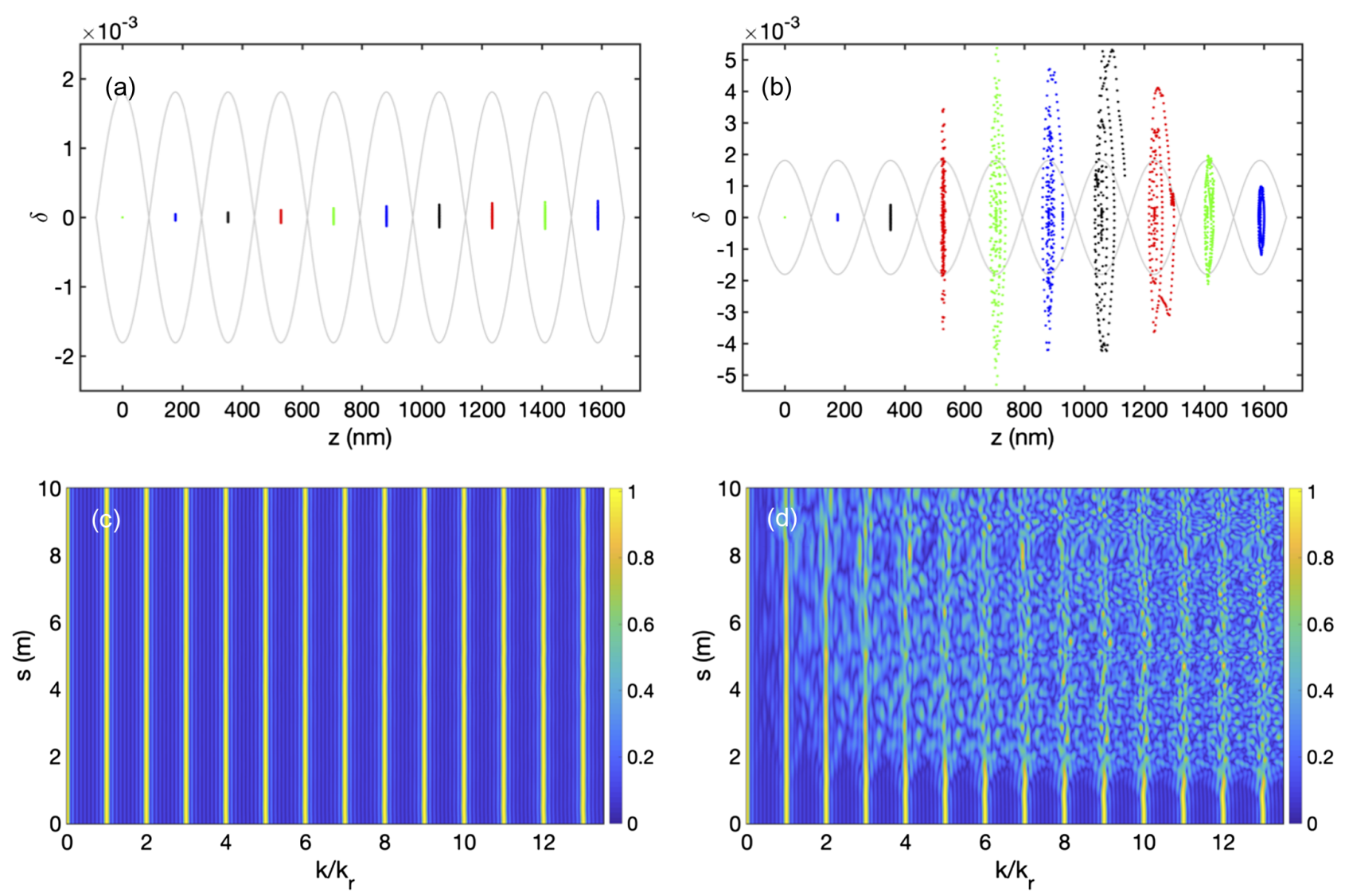

FIG. 11. (a,b) Macroparticle phase space evolution for individual microbunches at $s=10 \mathrm{~m}$ with different microbunch charges; (c,d) Evolution of the bunching spectrum along the undulator as a function of the normalized wavenumber. Starting from the $n=0$ microbunch, the individual microbunch charges for the left column assume an increasing sequence $(1,2,3, \ldots, 10) \times 1.2 \times 10^{5}$, while for the right column a decreasing sequence $(10,9,8, \ldots, 1) \times 1.2 \times 10^{5}$. See the context for more discussions.

e.g., an array of $N_{\mathrm{pb}}=(1,2,3, \ldots, 10) \times 1.2 \times 10^{5}$ for $n=0,1,2, \ldots 10$. The evolutions of the macroparticle phase space portrait and the bunching spectrum are shown in Figs. 11(a) and 11(c), respectively. Another sequence of the reverse order for the microbunch charges, $N_{\mathrm{pb}}=$ $(10,9,8, \ldots, 1) \times 1.2 \times 10^{5}$, is also simulated and shown in Figs. 11(b) and 11(d). A dramatic difference between the two cases again indicates that the coherent undulator radiation does not only disturb the leading microbunches but also provide additional focusing. The undulator radiation fields tend to more disturb the leading microbunches than to provide focusing. Therefore, the microbunch near behind should have lower charges to ensure the perturbation does not destroy the test microbunches, thus encouraging increasing microbunch charge sequence. Here, we note that the microbunch charge sequence is not optimized; optimization of the sequence is not of our current interest.

One may wonder about the multibunch dynamics when the microbunch spacing deviates from the undulator resonant wavelength. Figure 12 shows the evolution of the macroparticle phase space portrait and the bunching spectrum for three different cases: $\Delta L=0.99 \lambda_{r}$ (left), $\Delta L=\lambda_{r}$ (middle), and $\Delta L=1.01 \lambda_{r}$ (right). Although the bunch space may not be exactly the resonant wavelength, the microbunch train remains equally spaced. It is interesting to find that when the bunch spacing is exactly the resonant wavelength, the individual microbunches gain an extra focusing and experience a weaker radiation field. The shorter or longer microbunch spacing deviates from the resonant wavelength, and thus the individual microbunches may no longer attain the additional focusing or experience a stronger radiation field. We will further elaborate this soon. The evident longitudinal oscillations from the resonant driving quickly degrade the bunching factor of the microbunch train. From the bottom row of Fig. 12 we may conclude that to ensure the bunching quality at $s=2 \mathrm{~m}$, the initial detuned bunch spacing must be within $0.5 \%$ or smaller. If the external laser can effectively and accurately phase lock the individual microbunches, the bunching factor can be well preserved.

To further elaborate the physical mechanism outlined above, Fig. 13 shows the coherent radiation wake function and its derivative, which is proportional to the induced potential-well term. See Eqs. (20) and (22) and note that the momentum compaction factor of the undulator is a negative value. The red, green, and blue dots respectively represent 

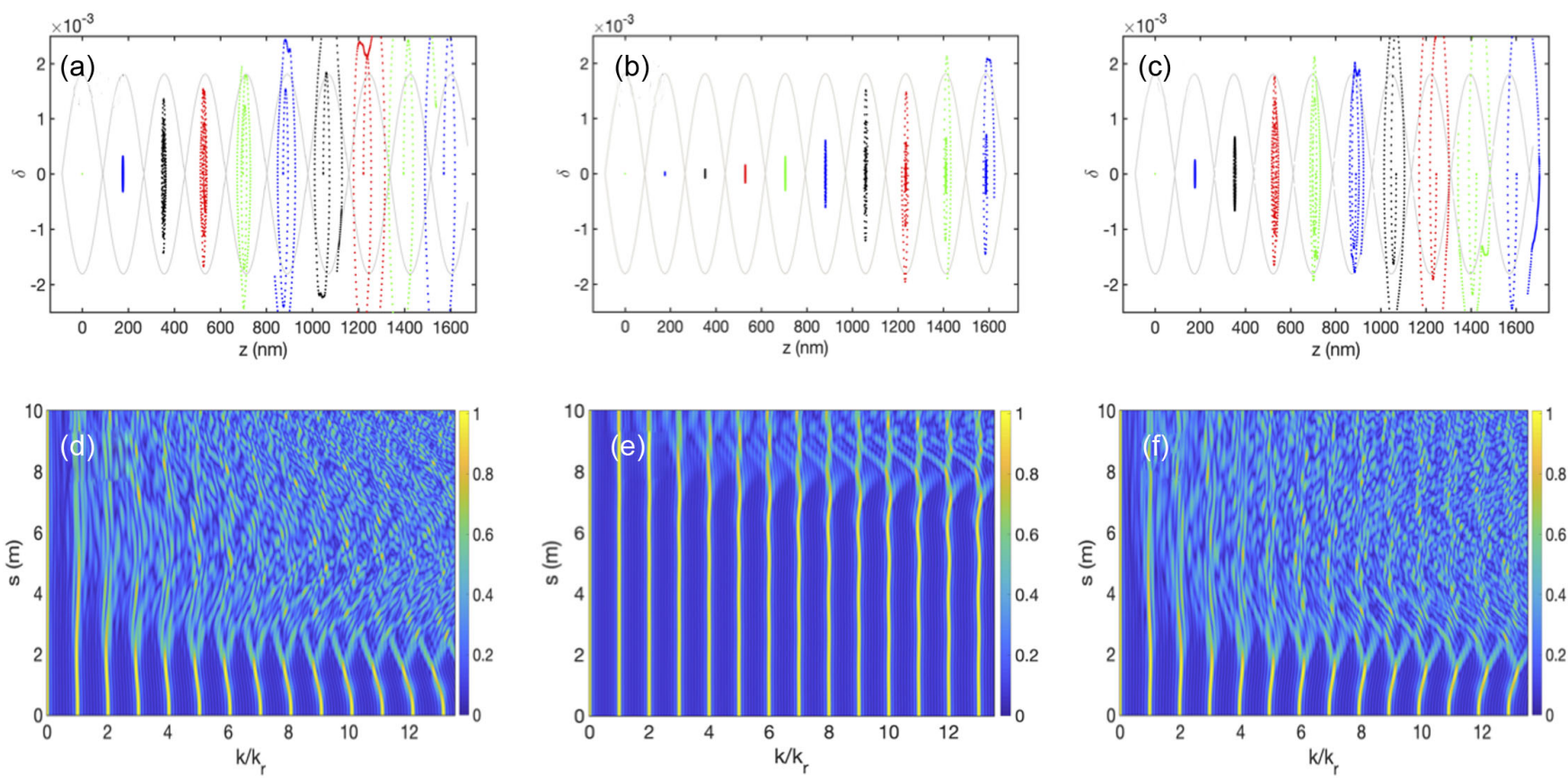

FIG. 12. (a,b,c) Macroparticle phase space evolution for individual microbunches at $s=10 \mathrm{~m}$ with different bunch spacings; (d,e,f) Evolution of the bunching spectrum along the undulator as a function of the normalized wavenumber. The microbunch spacing for the left column assumes $\Delta L=0.99 \lambda_{r}$, the central column $\Delta L=\lambda_{r}$, and the right column $\Delta L=1.01 \lambda_{r}$. For the three cases, the bunch spacing is uniform for all microbunches. See the context for more discussions.

the microbunch trains with $\Delta L=\lambda_{r}, \Delta L=0.99 \lambda_{r}$, and $\Delta L=1.01 \lambda_{r}$. One can see from the figure that when the bunch spacing is equal to the resonant wavelength, i.e., the red dots, the individual microbunches are located around the bottom of the potential well. When the bunching spacing deviates from the ideal case, the more leading microbunches deviate further from the bottom of the well and experience a stronger radiation fields (dashed line).

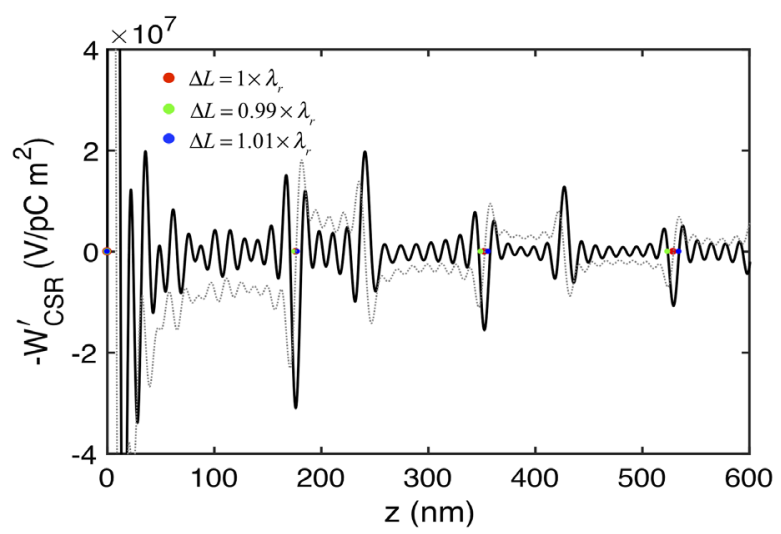

FIG. 13. Illustration of the coherent undulator radiation wake function (dashed line), its derivative (solid line), and a few microbunches for different bunch spacings. See the context for more discussions. The wake function is scaled to help visualization.
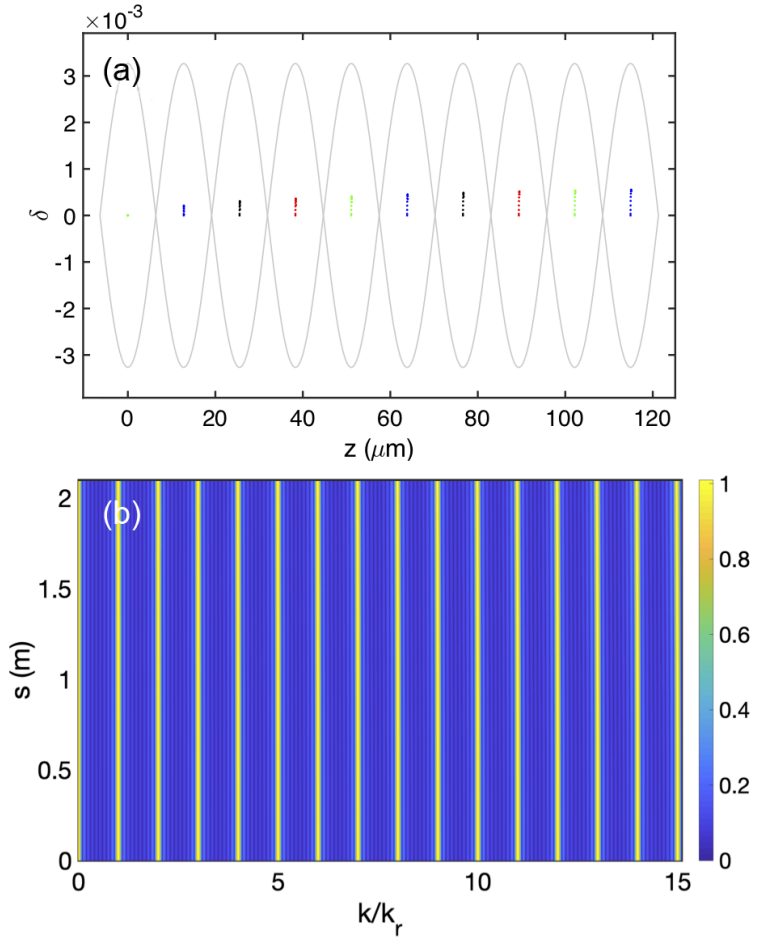

FIG. 14. Macroparticle phase space evolution for individual microbunches at $s=2 \mathrm{~m}$; (b) Evolution of the bunching spectrum along the undulator. Here $H_{\max }=15$ and $z_{n}(0)=0$ for all macroparticles. 


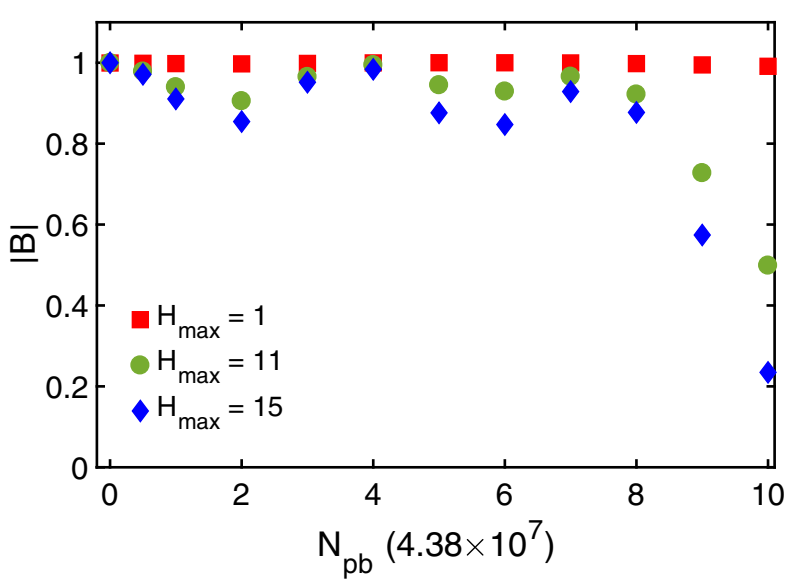

FIG. 15. Dependence of the bunching factors $|\mathcal{B}|$ on the number of electrons per microbunch including different radiation harmonics. The bunching factor is evaluated at $s=2.1 \mathrm{~m}$.

\section{B. Example 2: IR}

Now we consider the second example, dubbed as IR. Different from the first example, the amount of the microbunch charge is now 400 times that of the EUV case, and the bunch spacing or the resonant wavelength is 50 times longer than that of the EUV case. The total length of the undulator is similar to that of the EUV case and the reference electron energy is the same. The undulator parameter $K$ and the undulator period are adjusted by the resonance condition [Eq. (3)] accordingly. Figures 14 and 15 show the numerical results based on the parameters in Table I. Due to a larger microbunch charge, if we assume the uncorrelated energy spread $\sigma_{\delta} \approx 10^{-3}$, the maximum harmonics may be set $H_{\max }=15$. By design, the microbunch train is aimed to radiate at the 11th harmonics in the radiator.

It can be seen from Fig. 14 that the bunching factor is well preserved along the undulator up to 15 th harmonics. The centroid offsets of the individual microbunches at the exit of the undulator are negligibly small compared with the bunch spacing $12.8 \mu \mathrm{m}$. Moreover from Fig. 15, we see that the bunching factor remains larger than 0.8 up to eight times the value of the electron numbers per microbunch.

\section{SUMMARY AND DISCUSSION}

In this paper, we have formulated the coherent-radiationinduced multibunch BBU instability for a steady-state microbunch train in an undulator based on the macroparticle model. From the undulator radiation spectrum, we obtained the corresponding coherent radiation wake function. We have then derived a set of macroparticle equations of motion for the individual microbunches and identified possible mechanisms when microbunch spacing is close to the undulator resonant wavelength. Since the radiation fields copropagate with the electron beam, the slippage effect must be included in the formulation and becomes important when the length of the undulator is short and the microbunch train is long. The relatively long-range wake field from spectral peaks around the integer multiples of undulator resonant frequencies can overtake the leading microbunches and result in resonant driving multibunch BBU instability. The bunching factor associated with the multibunch oscillations is used to quantify how well the microbunching is preserved when the beam traverses the undulator. Our developed theoretical formulation is applied to two SSMB design examples, where we find that, when the bunch spacing is close to the undulator resonant wavelength, the coherent radiation can provide extra longitudinal focusing for the near leading microbunches, while the fields tend to perturb the far leading microbunches. For the specific scenario considered in this paper, this mechanism results in more stable multibunch oscillations. For the given parameters of the two examples, the multibunch oscillations remain stable in the $2 \mathrm{~m}$ long undulator. To better illustrate the multibunch dynamics, we have increased the number of electrons per microbunch for one to two orders of magnitude compared with the preliminary design parameters [41]. For the real SSMB design with the average beam current of $1 \mathrm{~A}$, the single-pass longitudinal BBU instability should not be so severe as that shown in the studied examples. This observation may shed light on more thorough beam dynamics studies of the longitudinal strong-focusing SSMB when the microbunch trains would circulate in a storage ring for multiple passes.

Here, we comment on some possible extensions of this work. As for construction of the radiation impedance, the adopted model is a free-space model [37,38], i.e., any possible shielding by vacuum pipes is not taken into consideration. Concerning the beam dynamics, using the macroparticles to represent the individual microbunches can be oversimplified. For individual microbunches, any possible position-energy correlation, i.e., energy chirp, is ignored, which may result in change of microbunch peak current and the dynamics in the undulator. The warm beam effects, including the finite energy spread and the transverse finite size/emittance, are also not included. More importantly, when the analysis would be applied to the multipass, storage-ring configuration, those diffusion effects should be taken into account, including intrabeam scattering (IBS) or/and synchrotron radiation damping and excitation. All these effects may be supplemented by Vlasov or FokkerPlanck formulation. Recent studies indicate that for the low- or mid-energy high-brightness electron beam, IBS may play a significant role in microbunching development even in a single-pass beam transport line [53-56]. As outlined in Sec. III, we have so far restricted ourselves to the single-pass multibunch dynamics in an undulator with an external modulation laser. Further extension to SSMB scenarios includes installation of the cavity mirrors to form 
a resonator-modulator system. Because the frequency of the coherent undulator radiation is close to that of the laser cavity resonator, the undulator radiation fields may be trapped in the cavity mirrors, bounce back and forth, and eventually affect the trailing microbunch train(s) when the fields do not significantly decay after the cavity round trip time. In this situation the multipass multibunch dynamics should be taken into consideration. The present formulation belongs to the time-domain analysis and thus suitable for single-pass dynamics. An analogy may be drawn in the long-term multipass situation to the Robinson instability based on frequency-domain analysis $[1,2]$. These possible extensions and discussions are in our working plan.

As a side note, we compare the coherent-radiationinduced beam instability with the short-wavelength FEL sideband instability [57-59]. First, for the FEL-microbunching process starting from the unbunched or coasting beam, the main signal [Eq. (3)] and the bunching factor exponentiate and stand out after ten power gain lengths [52]. For the scenario discussed in this paper, the main signal originates from the coherent radiation when the microbunch train traverses an undulator. The bunching factor can be well preserved with proper longitudinal focusing provided by the external laser and the coherent radiation field. The radiation fields in principle grow quadratically along $s$ in the undulator [60]. Second, when reaching FEL saturation, the significant laser field and the undulator magnetic field form the phase space bucket, where the gradually microbunched beam begin to execute synchrotron oscillation. Such synchrotron motion results in the FEL sideband signal. From existing FEL sideband theories [61], when the main signal is assumed at saturation, the sideband signal would grow exponentially. With proper undulator tapering, the main signal continues to grow and may effectively suppress sideband instability [59]. The effect of transverse radiation diffraction is also found to mitigate the sideband signals $[58,62,63]$. For the scenario considered in this paper, the undulator is untapered and is designed to be a small fraction of the FEL power gain length to avoid energy heating. Should this multibunch instability (analogous to FEL sideband instability) occur, a proper undulator tapering can be an option for possible mitigation. Finally, for the FEL the (saturated) main signal, together with the undulator field, is considered as a driving source for the sideband instability. In our situation, the longitudinal phase space bucket is initially formed by the external laser and the undulator magnetic field. The individual microbunches execute synchrotron oscillations and are gradually perturbed by the trailing microbunches. The additional focusing can be provided by the near behind microbunches when the bunch spacing is close to the resonant wavelength. Overall this beneficial effect would favor the phase-locked bunch spacing from the dedicated external laser system.

\section{ACKNOWLEDGMENTS}

One of the authors (C. T.) acknowledges S. Lau for help with the preparation of this manuscript. He would also like to thank Xiujie Deng (Tsinghua University) and Weilun Qin (DESY) for many insightful discussions on this work and helpful comments regarding this manuscript. This work is supported by the Fundamental Research Funds for the Central Universities under Project No. 5003131049 and National Natural Science Foundation of China under Project No. 11905073.

[1] A. W. Chao, Physics of Collective Beam Instabilities in High Energy Accelerators (John Wiley \& Sons, New York, 1993).

[2] K.-Y. Ng, Physics of Intensity Dependent Beam Instabilities (World Scientific, Singapore, 2006).

[3] S. A. Heifets and S. A. Kheifets, Coupling impedance in modern accelerators, Rev. Mod. Phys. 63, 631 (1991).

[4] W. K. H. Panofsky and R. B. Neal, Electrons accelerated to the 10-to 20-GeV range, Science 152, 1353 (1966).

[5] W. K. H. Panofsky and M. Bander, Asymptotic theory of beam break-up in linear accelerators, Rev. Sci. Instrum. 39, 206 (1968).

[6] A. W. Chao, B. Richter, and C.-Y. Yao, Beam emittance growth caused by transverse deflecting fields in a linear accelerator, Nucl. Instrum. Methods Phys. Res., Sect. A 178, 1 (1980).

[7] K. A. Thompson and R. D. Ruth, Transverse and longitudinal coupled bunch instabilities in trains of closely spaced bunches, in Proceedings of the 1989 IEEE Particle Accelerator Conference, Accelerator Science and Technology (IEEE, Chicago, 1989), pp. 792-794, https:// accelconf.web.cern.ch/p89/PDF/PAC1989_0792.PDF.

[8] R. D. Kohaupt, Simplified presentation of head tail turbulence, DESY Report, pp. 80-19, 1980.

[9] R. Talman, The influence of finite synchrotron oscillation frequency on the transverse head-tail effect, Nucl. Instrum. Methods Phys. Res., Sect. A 193, 423 (1982).

[10] E. L. Saldin, E. A. Schneidmiller, and M. V. Yurkov, Klystron instability of a relativistic electron beam in a bunch compressor, Nucl. Instrum. Methods Phys. Res., Sect. A 490, 1 (2002).

[11] S. Heifets, G. Stupakov, and S. Krinsky, Coherent synchrotron radiation instability in a bunch compressor, Phys. Rev. ST Accel. Beams 5, 064401 (2002).

[12] Z. Huang and K.-J. Kim, Formulas for coherent synchrotron radiation microbunching in a bunch compressor chicane, Phys. Rev. ST Accel. Beams 5, 074401 (2002).

[13] M. Venturini, Microbunching instability in single-pass systems using a direct two-dimensional Vlasov solver, Phys. Rev. ST Accel. Beams 10, 104401 (2007).

[14] M. Venturini, R. Warnock, and A. Zholents, Vlasov solver for longitudinal dynamics in beam delivery systems for X-ray free electron lasers, Phys. Rev. ST Accel. Beams 10, 054403 (2007).

[15] M. Venturini, R. Warnock, R. Ruth, and J. A. Ellison, Coherent synchrotron radiation and bunch stability in a 
compact storage ring, Phys. Rev. ST Accel. Beams 8, 014202 (2005).

[16] G. Stupakov and S. Heifets, Beam instability and microbunching due to coherent synchrotron radiation, Phys. Rev. ST Accel. Beams 5, 054402 (2002).

[17] S. Heifets and G. Stupakov, Single-mode coherent synchrotron radiation instability, Phys. Rev. ST Accel. Beams 6, 064401 (2003).

[18] S. Heifets, Single-mode coherent synchrotron radiation instability of a bunched beam, Phys. Rev. ST Accel. Beams 6, 080701 (2003).

[19] C.-Y. Tsai, D. Douglas, R. Li, and C. Tennant, Linear microbunching analysis for recirculation machines, Phys. Rev. Accel. Beams 19, 114401 (2016).

[20] C.-Y. Tsai, Ya. S. Derbenev, D. Douglas, R. Li, and C. Tennant, Vlasov analysis of microbunching instability for magnetized beams, Phys. Rev. Accel. Beams 20, 054401 (2017).

[21] Ya. S. Derbenev, J. Rossbach, E. L. Saldin, and V. D. Shiltsev, Microbunch radiative tail-head interaction, DESY Print, TESLA-FEL, pp. 95-05, 1995.

[22] J. B. Murphy, R. L. Gluckstern, and S. Krinsky, Longitudinal wake field for an electron moving on a circular orbit, Part. Accel. 57, 9 (1996), https://cds.cern.ch/record/ 1120287/files/p9.pdf.

[23] E. L. Saldin, E. A. Schneidmiller, and M. V. Yurkov, On the coherent radiation of an electron bunch moving in an arc of a circle, Nucl. Instrum. Methods Phys. Res., Sect. A 398, 373 (1997).

[24] T. Agoh and K. Yokoya, Calculation of coherent synchrotron radiation using mesh, Phys. Rev. ST Accel. Beams 7, 054403 (2004).

[25] C. Mitchell, J. Qiang et al., Effects of transient CSR wakefields on microbunching in a bunch compressor, in Proceedings of the 4th International Particle Accelerator Conference, Shanghai, China, TUPWA057 (2013), https:// accelconf.web.cern.ch/IPAC2013/papers/tupwa057.pdf.

[26] D. Zhou et al., An alternative 1D model for CSR with chamber shielding, in Proceedings of the 3rd International Particle Accelerator Conference, New Orleans, Louisiana, USA, MOOBB03 (2012), https://accelconf.web.cern.ch/ ipac2012/papers/moobb03.pdf.

[27] R. Li and C.-Y. Tsai, CSR impedance for nonultrarelativistic beams, in Proceeeings of the 6th International Particle Accelerator Conference, Richmond, VA, USA, MOPMN004 (2015), https://accelconf.web.cern .ch/IPAC2015/papers/mopmn004.pdf.

[28] R. Li and C.-Y. Tsai, Entrance and exit CSR impedance for non-ultrarelativistic beam, in Proceedings of IPAC2017, Copenhagen, Denmark, WEPIK113 (2017), https:// accelconf.web.cern.ch/ipac2017/papers/wepik113.pdf.

[29] G. Stupakov and D. Zhou, Analytical theory of coherent synchrotron radiation wakefield of short bunches shielded by conducting parallel plates, Phys. Rev. Accel. Beams 19, 044402 (2016).

[30] C.-Y. Tsai, S. Di Mitri, D. Douglas, R. Li, and C. Tennant, Conditions for coherent-synchrotron-radiation-induced microbunching suppression in multibend beam transport or recirculation arcs, Phys. Rev. Accel. Beams 20, 024401 (2017).
[31] D. R. Douglas, S. V. Benson, A. Hutton, G. A. Krafft, R. Li, G. R. Neil, Y. Roblin, C. D. Tennant, and C.-Y. Tsai, Control of coherent synchrotron radiation and microbunching effects during transport of high brightness electron beams, arXiv:1403.2318.

[32] D. Douglas, S. Benson, A. Hofler, R. Kazimi, G. Krafft, R. Li, Y. Roblin, C. Tennant, B. Terzifa, and C.-Y. Tsai, Control of synchrotron radiation effects during recirculation, in Proceedings of the 6th International Particle Accelerator Conference, Richmond, VA, USA, TUPMA038 (2015), https://accelconf.web.cern.ch/ ipac2015/papers/tupma035.pdf.

[33] D. Douglas, S. Benson, R. Li, Y. Roblin, C. Tennant, G. Krafft, B. Terzic, and C. Tsai, Control of synchrotron radiation effects during recirculation with bunch compression, in Proceedings of the 6th International Particle Accelerator Conference, Richmond, VA, USA, TUPMA034 (2015), https://accelconf.web.cern.ch/ ipac2015/papers/tupma034.pdf.

[34] D. F. Alferov, Yu. A. Bashmakov, and P. A. Cherenkov, Radiation from relativistic electrons in a magnetic undulator, Sov. Phys. Usp. 32, 200 (1989).

[35] S. Krinsky, M. L. Perlman, and R. E. Watson, Characteristics of synchrotron radiation and of its sources, Technical report No. BNL-27678, Brookhaven National Lab., Upton, NY (USA), 1979, https://digital.library.unt.edu/ark:/ 67531/metadc1068000/.

[36] A. Hofmann, The Physics of Synchrotron Radiation, Vol. 20 (Cambridge University Press, Cambridge, England, 2004).

[37] E. L. Saldin, E. A. Schneidmiller, and M. V. Yurkov, Radiative interaction of electrons in a bunch moving in an undulator, Nucl. Instrum. Methods Phys. Res., Sect. A 417, 158 (1998).

[38] J. Wu, T. O. Raubenheimer, and G. V. Stupakov, Calculation of the coherent synchrotron radiation impedance from a wiggler, Phys. Rev. ST Accel. Beams 6, 040701 (2003).

[39] J. Wu, G. V. Stupakov, T. O. Raubenheimer, and Z. Huang, Impact of the wiggler coherent synchrotron radiation impedance on the beam instability and damping ring optimization, Phys. Rev. ST Accel. Beams 6, 104404 (2003).

[40] A. W. Chao, Lectures on Accelerator Physics, (World Scientific, Singapore, 2020).

[41] A. Chao, E. Granados, X. B. Huang, D. Ratner, and H.-W. Luo, High power radiation sources using the steady-state microbunching mechanism, in Proceedings of 7th International Particle Accelerator Conference, Busan, Korea IPAC16, TUXB01 (2016).

[42] A. Chao et al., A compact high-power radiation source based on steady-state microbunching mechanism, Technical Report No. SLAC-PUB-17241, Stanford Linear Accelerator Center (SLAC), 2018, https://www.slac.stanford .edu/pubs/slacpubs/17000/slac-pub-17241.pdf.

[43] Y. Jiao, D. F. Ratner, and A. W. Chao, Terahertz coherent radiation from steady-state microbunching in storage rings with x-band radio-frequency system, Phys. Rev. ST Accel. Beams 14, 110702 (2011).

[44] C. Tang, X. Deng, W. Huang, T. Rui, A. Chao, J. Feikes, J. Li, M. Ries, C. Feng, B. Jiang et al., An overview of the progress on SSMB, in Proceedings of the 60th ICFA 
Advanced Beam Dynamics Workshop on Future Light Sources, Shanghai (ICFA Advanced Beam Dynamics Workshop, 2018), https://accelconf.web.cern.ch/fls2018/ papers/thp2wb02.pdf.

[45] D. F. Ratner and A. W. Chao, Steady-State Microbunching in a Storage Ring for Generating Coherent Radiation, Phys. Rev. Lett. 105, 154801 (2010).

[46] X. J. Deng, A. W. Chao, J. Feikes, W. H. Huang, M. Ries, and C. X. Tang, Single-particle dynamics of microbunching, Phys. Rev. Accel. Beams 23, 044002 (2020).

[47] X. J. Deng, R. Klein, A. W. Chao, A. Hoehl, W. H. Huang, J. Li, J. Lubeck, Y. Petenev, M. Ries, I. Seiler et al., Widening and distortion of the particle energy distribution by chromaticity in quasi-isochronous rings, Phys. Rev. Accel. Beams 23, 044001 (2020).

[48] X. Deng, A. Chao, J. Feikes, A. Hoehl, W. Huang, R. Klein, A. Kruschinski, J. Li, A. Matveenko, Y. Petenev et al., Experimental demonstration of the mechanism of steadystate microbunching, Nature (London) 590, 576 (2021).

[49] J. D. Jackson, Classical Electrodynamics (John Wiley \& Sons, New York, 2007).

[50] H. Wiedemann, Particle Accelerator Physics, 4th ed. (Springer Nature, New York, 2015).

[51] K.-J. Kim, Characteristics of synchrotron radiation, in AIP Conference Proceedings, Vol. 184 (American Institute of Physics, New York, 1989), pp. 565-632.

[52] K.-J. Kim, Z. Huang, and R. Lindberg, Synchrotron Radiation and Free-Electron Lasers: Principles of Coherent X-Ray Generation (Cambridge university press, Cambridge, England, 2017).

[53] G. Stupakov et al., Effect of coulomb collisions on echoenabled harmonic generation (EEHG), in Proceedings of 33rd International Free Electron Laser Conference 2011, WEPSO68, Vol. 49 (2011), https://accelconf.web.cern.ch/ FEL2011/papers/mopb20.pdf.

[54] G. Penn, Intra-beam scattering for free electron lasers and its modeling in chicanes, LBL Report, 2014.
[55] C.-Y. Tsai, W. Qin, K. Fan, X. Wang, J. Wu, and G. Zhou, Theoretical formulation of phase space microbunching instability in the presence of intrabeam scattering for single-pass or recirculation accelerators, Phys. Rev. Accel. Beams 23, 124401 (2020).

[56] C.-Y. Tsai and W. Qin, Semi-analytical analysis of highbrightness microbunched beam dynamics with collective and intrabeam scattering effects, Phys. Plasmas 28, 013112 (2021).

[57] C.-Y. Tsai, J. Wu, C. Yang, M. Yoon, and G. Zhou, Sideband instability analysis based on a one-dimensional high-gain free electron laser model, Phys. Rev. Accel. Beams 20, 120702 (2017).

[58] C.-Y. Tsai, J. Wu, C. Yang, M. Yoon, and G. Zhou, Singlepass high-gain tapered free-electron laser with transverse diffraction in the postsaturation regime, Phys. Rev. Accel. Beams 21, 060702 (2018).

[59] C.-Y. Tsai, C. Emma, J. Wu, M. Yoon, X. Wang, C. Yang, and G. Zhou, Area-preserving scheme for efficiency enhancement in single-pass tapered free electron lasers, Nucl. Instrum. Methods Phys. Res., Sect. A 913, 107 (2019).

[60] L. H. Yu and J. Wu, Theory of high gain harmonic generation: An analytical estimate, Nucl. Instrum. Methods Phys. Res., Sect. A 483, 493 (2002).

[61] N. Kroll, P. Morton, and M. Rosenbluth, Free-electron lasers with variable parameter wigglers, IEEE J. Quantum Electron. 17, 1436 (1981).

[62] Y. Jiao, J. Wu, Y. Cai, A. W. Chao, W. M. Fawley, J. Frisch, Z. Huang, H.-D. Nuhn, C. Pellegrini, and S. Reiche, Modeling and multidimensional optimization of a tapered free electron laser, Phys. Rev. ST Accel. Beams 15, 050704 (2012).

[63] C. Emma, K. Fang, J. Wu, and C. Pellegrini, High efficiency, multiterawatt x-ray free electron lasers, Phys. Rev. Accel. Beams 19, 020705 (2016). 